\title{
THE COINCIDENCE OF NUCLEAR STAR CLUSTERS AND ACTIVE GALACTIC NUCLEI
}

\author{
ANIL SETH $^{1}$ \\ Harvard-Smithsonian Center for Astrophysics, Cambridge, MA 02138; aseth@cfa.harvard.edu \\ AND \\ Marcel Agüeros, ${ }^{2}$ Duane Lee, and Antara Basu-Zych \\ Columbia University, New York, NY 10027 \\ Received 2007 October 26; accepted 2007 December 20
}

\begin{abstract}
We study galaxies that host both nuclear star clusters and AGNs, implying the presence of a massive black hole. We select a sample of 176 galaxies with previously detected nuclear star clusters that range from ellipticals to late-type spirals. We search for AGNs in this sample using optical spectroscopy and archival radio and X-ray data. We find galaxies of all Hubble types and with a wide range of masses $\left(10^{9}-10^{11} M_{\odot}\right)$ hosting both AGNs and nuclear star clusters. From the optical spectra, we classify $10 \%$ of the galaxies as AGN and an additional $15 \%$ as composite, indicating a mix of AGN and star formation spectra. The fraction of nucleated galaxies with AGNs increases strongly as a function of galaxy and nuclear star cluster mass. For galaxies with both a nuclear star cluster and a black hole, we find that the masses of these two objects are quite similar. However, nondetections of black holes in Local Group nuclear star clusters show that not all clusters host black holes of similar masses. We discuss the implications of our results for the formation of nuclear star clusters and massive black holes.
\end{abstract}

Subject headings: galaxies: active — galaxies: formation — galaxies: nuclei — galaxies: star clusters

Online material: machine-readable table

\section{INTRODUCTION}

Nuclear star clusters (NCs) are massive star clusters coincident with the photocenters of galaxies. They are very common and have been found in $\sim 75 \%$ of local late-type spirals (Böker et al. 2002) and Virgo dwarf elliptical galaxies (Côté et al. 2006). Their size is similar to that of globular clusters (Böker et al. 2004), but NCs are 1-2 orders of magnitude brighter and more massive (Walcher et al. 2005). Also, unlike most globular clusters, they have extended star formation histories (Walcher et al. 2006; Rossa et al. 2006) and complex morphologies (Seth et al. 2006).

The luminosity of NCs correlates with galaxy luminosity in both ellipticals (Lotz et al. 2001; Graham \& Guzmán 2003; Côté et al. 2006) and spirals (Carollo et al. 1998; Böker et al. 2004). Recently, it has been shown that the masses of NCs follow scaling relationships with galaxy mass $\left(M_{\mathrm{gal}}\right)$, bulge velocity dispersion $(\sigma)$, and Sérsic index (Ferrarese et al. 2006; Wehner \& Harris 2006; Rossa et al. 2006; Graham \& Driver 2007). These scaling relations are very similar to those seen for massive black holes $(\mathrm{MBHs}){ }^{3}$ appearing to extend those relations to lower masses. Thus, Wehner \& Harris (2006) and Ferrarese et al. (2006) suggest that there may be a single scaling relation linking the mass of a central massive object (CMO; either an $\mathrm{NC}$ or $\mathrm{MBH}$ ) to the large-scale properties of the galaxy. The existence of an $M_{\mathrm{CMO}}-\sigma$ or $M_{\mathrm{CMO}}-M_{\text {gal }}$ relation suggests that the formation of the $\mathrm{CMO}$ is linked in some way to the evolution of the galaxy. Theoretically, these scaling relationships can be understood in multiple ways. They could be created by feedback from either NCs or MBHs regulating the star formation in the galaxy as a whole

\footnotetext{
1 CfA Fellow.

2 NSF Astronomy and Astrophysics Postdoctoral Fellow.

3 We use MBHs to refer to all non-stellar-mass black holes, including those normally referred to as intermediate-mass and supermassive black holes.
}

(e.g., McLaughlin et al. 2006b), or alternatively, they could simply result from gas accretion onto the nucleus in proportion to the galaxies' mass (e.g., Li et al. 2007).

Despite this interesting connection between NCs and MBHs and their link to galaxy formation and evolution, no systematic study of the overlap between these classes of objects exists. A handful of objects, including the Milky Way, are already known to host both NCs and MBHs (see $\S 4$ ). Ferrarese et al. (2006) and Wehner \& Harris (2006) show that there is a rough transition at galaxy masses of $\sim 10^{10} M_{\odot}$ (and corresponding CMO mass of $\sim 10^{7} M_{\odot}$ ), above which galaxies typically host MBHs, and below which galaxies have NCs. While there is good evidence that more massive galaxies do not in fact host NCs (Côté et al. 2006), it remains unclear how common MBHs are in lower mass galaxies (e.g., Greene \& Ho 2007). Recent theoretical work shows that MBHs could form from stellar mergers in a young, dense cluster environment (Miller \& Colbert 2004; Portegies Zwart et al. 2004), and a direct link between NC and MBH formation may therefore exist.

We present a systematic study of the overlap between NCs and MBHs aimed at better understanding the relation between the two types of objects and the formation mechanism of CMOs in general. Starting with a sample of galaxies with known NCs, we search for active galactic nuclei (AGNs) that are powered by accretion onto an $\mathrm{MBH}$. This study gives a lower limit on the number of systems with MBHs, since quiescent and heavily obscured MBHs will not be detected as AGNs.

We begin by describing our sample of galaxies with NCs, drawn from several different catalogs (§ 2). Using optical spectra and radio and X-ray data, we examine our sample galaxies for evidence of AGN activity ( $(3)$. We then review galaxies for which detections of both AGNs or MBHs and NCs exist in the literature (§ 4). We discuss the demographics of galaxies with AGNs and NCs, the relative masses of these CMOs in galaxies where they 
TABLE 1

Sample of Galaxies with Nuclear Star Clusters

\begin{tabular}{|c|c|c|c|c|c|c|c|c|c|}
\hline Galaxy & Type & $\mathrm{T}$ & $\begin{array}{c}D \\
(\mathrm{Mpc})\end{array}$ & $M_{B}$ & $\log \left(M_{\mathrm{gal}} / M_{\odot}\right)$ & Source & $\begin{array}{l}\mathrm{NC} r_{\mathrm{eff}}^{\mathrm{a}} \\
(\operatorname{arcsec})\end{array}$ & $\log \left(M_{\mathrm{NC}} / M_{\odot}\right)$ & Spectra $^{\mathrm{b}}$ \\
\hline NGC $275 \ldots \ldots \ldots \ldots \ldots$ & $\mathrm{SBc}$ & 6.0 & 24.0 & -19.1 & $\ldots$ & B02 & 0.05 & 6.3 & None \\
\hline NGC $289 \ldots \ldots \ldots \ldots . . . .$. & $\mathrm{SBbc}$ & 3.9 & 27.4 & -20.5 & 10.9 & $\mathrm{C} 02$ & 0.13 & 7.9 & None \\
\hline NGC $300 \ldots \ldots \ldots \ldots . . . .$. & Scd & 6.9 & 1.9 & -17.6 & 9.3 & B02 & 0.13 & 6.0 & None \\
\hline NGC $337 \mathrm{a} . . . . . . . . . . .$. & SABd & 7.9 & 14.3 & -17.9 & 9.4 & B02 & 0.06 & 5.3 & None \\
\hline NGC $406 \ldots \ldots \ldots \ldots . . .$. & $\mathrm{Sc}$ & 4.9 & 17.5 & -18.2 & 9.7 & $\mathrm{C} 02$ & 0.10 & 5.8 & None \\
\hline NGC $428 \ldots \ldots \ldots \ldots . . . .$. & $\mathrm{SABm}$ & 8.6 & 15.9 & -19.1 & 9.8 & B02 & 0.05 & 6.5 & HFS97 \\
\hline NGC $450 \ldots \ldots \ldots \ldots . . . .$. & $\mathrm{SABc}$ & 5.9 & 24.4 & -19.2 & 9.7 & B02 & 0.11 & 6.1 & SDSS \\
\hline NGC $600 \ldots \ldots \ldots \ldots . . . . .$. & Scd & 6.9 & 25.1 & -19.0 & 9.9 & B02 & 0.06 & 6.2 & None \\
\hline NGC $853 \ldots \ldots \ldots \ldots . . . .$. & $\mathrm{Sm}$ & 8.7 & 21.0 & -15.9 & 9.6 & B02 & 0.05 & 6.1 & None \\
\hline NGC $986 \ldots \ldots \ldots \ldots . . . . .$. & $\mathrm{Sab}$ & 2.3 & 24.9 & -20.3 & 10.9 & $\mathrm{C} 02$ & 0.11 & 7.9 & None \\
\hline
\end{tabular}

Notes.-Table 1 is published in its entirety in the electronic edition of the Astrophysical Journal. A portion is shown here for guidance regarding its form and content.

a Asterisks indicate that NCs were unresolved by HST observations and thus have $r_{\text {eff }} \lesssim 0.03^{\prime \prime}$

b "S\&H" refers to spectra present in both SDSS and HFS97.

References.-(B02) Böker et al. 2002; (C97) Carollo et al. 1997; (C99) Carollo et al. 1998; (C02) Carollo et al. 2002; (C06) Côté et al. 2006; (S06) Seth et al. 2006.

coexist, and the implications of this study for CMO formation in $\S 5$. We conclude and discuss future work in $\S 6$.

\section{SAMPLE SELECTION AND PROPERTIES}

We have used catalogs from multiple studies to create a sample of nearby galaxies with known NCs. This sample, which contains galaxies of all Hubble types, is the starting point for finding galaxies that contain both NCs and MBHs.

The Hubble Space Telescope (HST) has enabled NCs to be identified in a large number of nearby galaxies with distances $\$ 30 \mathrm{Mpc}$. The use of HST is important both for distinguishing the NCs amid the crowded inner regions of galaxies and for resolving the clusters. In the studies used in our sample, the NCs have been selected from broadband optical or NIR images and are seen as compact sources distinct from the underlying galaxy profile. In the vast majority of the selected galaxies, the NCs have been resolved, suggesting that they are stellar sources and not AGN emission (see discussion in Rossa et al. 2006). Spectral studies of a number of the NCs in our sample confirm that their optical spectrum is dominated by starlight (Walcher et al. 2006; Rossa et al. 2006).

We use the following catalogs of galaxies with NCs:

1. Elliptical and lenticular galaxies from the ACS Virgo Cluster Survey of Côté et al. (2006). This survey includes both giant and dwarf elliptical galaxies in Virgo. Of the 100 elliptical galaxies in the survey, 51 contain NCs with measured properties (type "Ia" nuclei in Table 1 of Côté et al. 2006). These galaxies are all fainter than $M_{B} \gtrsim-19$; some brighter galaxies have apparent NCs, but Côté et al. (2006) are unable to derive their properties. The brightest galaxies in their sample $\left(M_{B}<-20.5\right)$ do not have any NCs. Of the $51 \mathrm{NCs}, 5$ are unresolved (see Table 1).

2. Early-type spiral galaxies from Carollo et al. (1997, 1998, 2002). Targets for their WFPC 2 and NICMOS snapshot programs are Sa-Sbc galaxies with $v_{\text {hel }}<2500 \mathrm{~km} \mathrm{~s}^{-1}$, angular diameter $>1^{\prime}$, and an inclination $<75^{\circ}$. Combining their WFPC2 and NICMOS studies, there are a total of 58 out of 94 galaxies with NCs; these are resolved in all but two cases.

3. Late-type spiral galaxies from Böker et al. (2002). This sample includes bulgeless spiral galaxies of type Scd-Sm, with $v_{\text {hel }}<2000 \mathrm{~km} \mathrm{~s}^{-1}$ and inclinations of $\lesssim 30^{\circ}$. Of 73 galaxies, 59 were found to have NCs.
4. Edge-on late-type ( $\mathrm{Sbc}-\mathrm{Scd})$ galaxies from Seth et al. (2006). Of the 14 galaxies in this sample, 9 have NCs, 6 of which are well resolved.

Our sample includes 176 galaxies with NCs spanning all galaxy types. Although the galaxy samples used are not complete, none of the selection criteria for these samples depend on the nuclear properties of the galaxies.

Galaxy distances were determined from a variety of sources, including using Virgo infall-corrected velocities from Hyperleda (Paturel et al. 2003) and NED1D ${ }^{4}$ for other distance indicators. For the Virgo Cluster galaxies, a distance of $16.5 \mathrm{Mpc}$ was assumed (Tonry et al. 2001; Mei et al. 2007). The galaxies range in distance between 2 and $40 \mathrm{Mpc}$, with most having distances between 10 and $30 \mathrm{Mpc}$. Galaxy magnitudes and Hubble types (including numerical types, " $T$ ") were also determined from Hyperleda. Figure 1 shows the type and absolute magnitude of all 176 galaxies in our sample. The $B$-band absolute magnitudes of these galaxies range between -15 and -21 . Galaxy masses were obtained using galaxy colors to estimate the $M / L$ ratios from Bell et al. (2003). We obtained optical color estimates for 147 of the 176 galaxies from Hyperleda (Paturel et al. 2003), including their $B-V$ colors, as well as colors from the Sloan Digital Sky Survey (SDSS; York et al. 2000) and 6dF survey (Jones et al. 2004). To obtain masses, we used both the Hyperleda total $B$ magnitudes corrected for internal and foreground extinction (also obtained from Hyperleda) and, where available, $K$-band magnitudes from the Two Micron All Sky Survey (2MASS) and DENIS. In cases where multiple sets of photometric data were available, the median value for the galaxy mass was used. The masses determined for a single galaxy using different methods typically differ by $\sim 35 \%(0.15 \mathrm{dex})$. The sample galaxy properties and distances are given in Table 1.

\subsection{Nuclear Star Cluster Masses}

The NCs in our sample have magnitudes measured in many different bands. To compare the NC properties for the entire sample, we therefore estimated the mass for each NC. There is strong evidence that NCs, at least in spiral galaxies, have complicated star formation histories (Walcher et al. 2006; Rossa et al. 2006;

\footnotetext{
${ }^{4}$ See http://nedwww.ipac.caltech.edu/level5/NED1D/.
} 


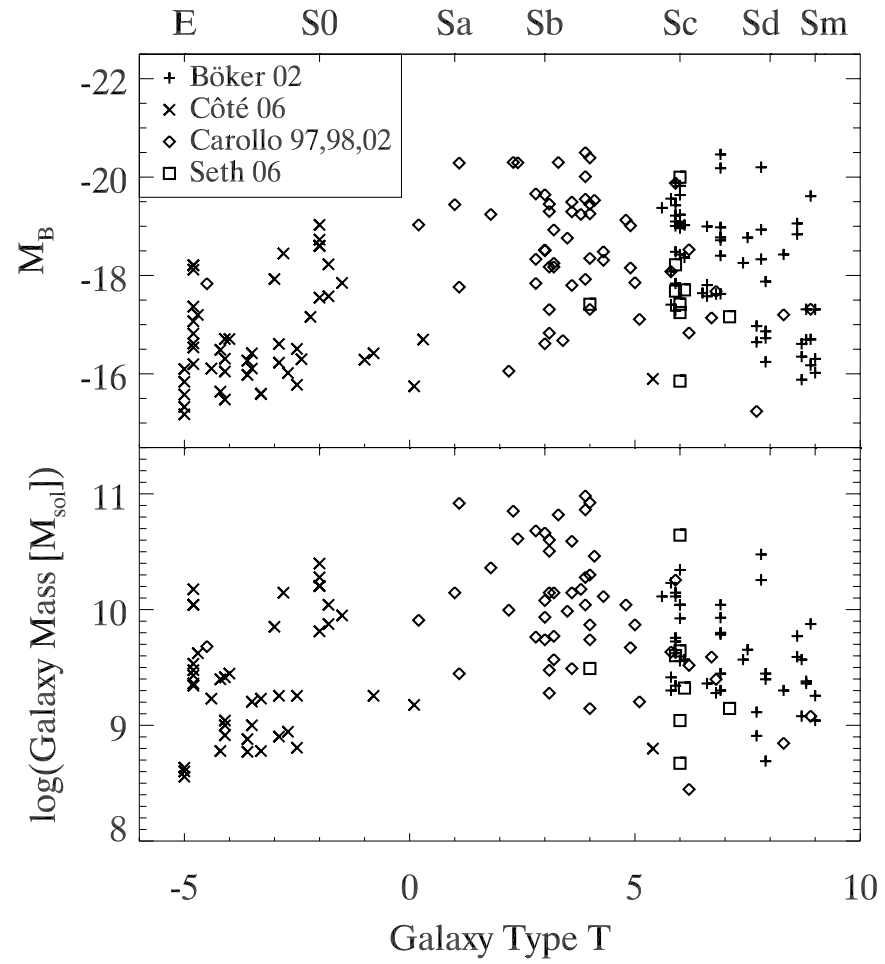

FIG. 1.-Top: Galaxy type and absolute magnitude of all 176 of our sample galaxies with known NCs. The $x$-axis gives the numerical galaxy type " $T$ " and corresponding Hubble type from Hyperleda (Paturel et al. 2003). Symbols indicate the source catalog for each galaxy as discussed in the text. Bottom: Galaxy mass for all 147 galaxies for which colors were available from Hyperleda.

Seth et al. 2006). Therefore, derivation of NC masses from integrated magnitudes is not straightforward. We have estimated the masses for the NCs in our sample using a variety of methods, as detailed below. The derived masses are shown in Table 1 and are used in determining the relative masses of NCs and MBHs and for examining the demographics of galaxies hosting both types of objects (see $\S 5)$ :

1. The best available mass estimates are dynamical measurements of nine NCs in the Böker sample by Walcher et al. (2005) and masses from population synthesis fits for an additional 15 spiral galaxies in Rossa et al. (2006).

2. For the Côté et al. (2006) clusters, we followed the prescription of Ferrarese et al. (2006), who estimate the NC mass by assuming an age of 5 Gyr and use the cluster's published $g-z$ color to determine the metallicity and thus the appropriate massto-light $(M / L)$ ratio from Bruzual \& Charlot (2003).

3. For the early-type spiral galaxies in the Carollo sample, we use the mean $B$-band $M / L$ ratio of $3.64 \pm 1.03$ derived for earlytype spiral NCs by Rossa et al. (2006). For most of the galaxies, both $V(\mathrm{WFPC} 2-\mathrm{F} 606 \mathrm{~W})$ and $H(\mathrm{NICMOS}-\mathrm{F} 160)$ magnitudes are available for each cluster, while a minority of galaxies have just one or the other magnitude available. We derive $B$-band magnitudes by assuming the colors of a single stellar population with $Z=0.030$ (matching the derived mean metallicity in Rossa et al. 2006) and age of 5.9 Gyr to match the $B$-band $M / L$ ratio (Bruzual \& Charlot 2003). These magnitudes were corrected for the foreground and estimated internal extinction as determined from Hyperleda (Paturel et al. 2003); the mean extinction correction was $A_{V}=0.27$.

4. For the late-type spiral galaxies, Walcher et al. (2005) have shown that the typical $I$-band $M / L$ ratio is $0.50 \pm 0.37$. We used this to derive masses from the $I$-band magnitudes in Böker et al. (2002) and Seth et al. (2006) after correcting for foreground and

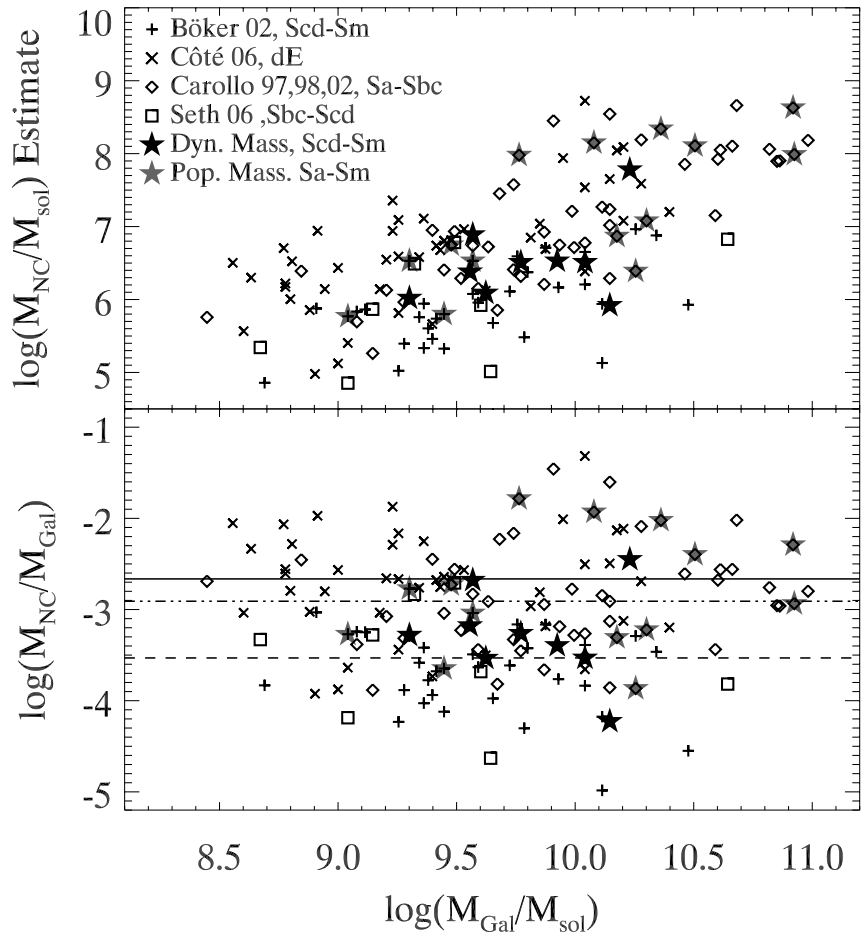

FIG. 2.-Top: NC mass vs. galaxy mass for all the clusters with derived galaxy masses in our sample. Bottom: Ratio of NC mass to galaxy mass. Overplotted are the median ratios for the elliptical galaxies (solid line), the early-type spirals (dotdashed line), and the late-type spirals (dashed line). In both panels the stars indicate dynamical masses measured for late-type spirals by Walcher et al. (2005; black stars) and the spectral synthesis masses from Rossa et al. (2006; gray stars).

internal extinction. We note the possibility that because of the selection of objects with bright apparent magnitudes, the Walcher et al. (2005) spectroscopic sample may not be representative of the Böker et al. (2002) sample as a whole. This may result in an underestimate of the $M / L$ ratio, as the study would favor younger, brighter NCs.

For the NCs with available dynamical masses (Walcher et al. 2005) or stellar population model masses (Rossa et al. 2006), the agreement between these masses and those obtained using the methods outlined above is good, with a mean difference of $-0.02 \pm$ 0.34 dex. This standard deviation of $0.34 \mathrm{dex}$ (factor of $\sim 2$ ) gives some indication of the error in our NC mass determinations.

Figure 2 shows the derived NC masses as a function of galaxy mass. Although correlations between $\mathrm{NC}$ and galaxy luminosities or masses have been shown for samples of galaxies of a single type (Carollo et al. 1998; Böker et al. 2004; Côté et al. 2006; Rossa et al. 2006; Ferrarese et al. 2006; Wehner \& Harris 2006), this is the first time they have been compared across all Hubble types. Figure 2 shows the expected correlation between NC mass and galaxy mass. However, we find an offset between earlier and later type galaxies, with the later type galaxies having less massive $\mathrm{NCs}$ at a given galaxy mass. This can be seen in the bottom panel of Figure 2, where we plot the ratio of NC to galaxy mass. Lines indicating the median NC to galaxy mass ratio for elliptical, earlytype spiral, and late-type spiral galaxies show that late-type spirals have NC masses about an order of magnitude below elliptical galaxies of the same mass. We will discuss these results in greater detail in a future paper.

\section{PRESENCE OF AGNs}

In this section we analyze the evidence for AGNs in our sample galaxies using optical spectroscopy $(\S 3.1)$ and radio and X-ray 


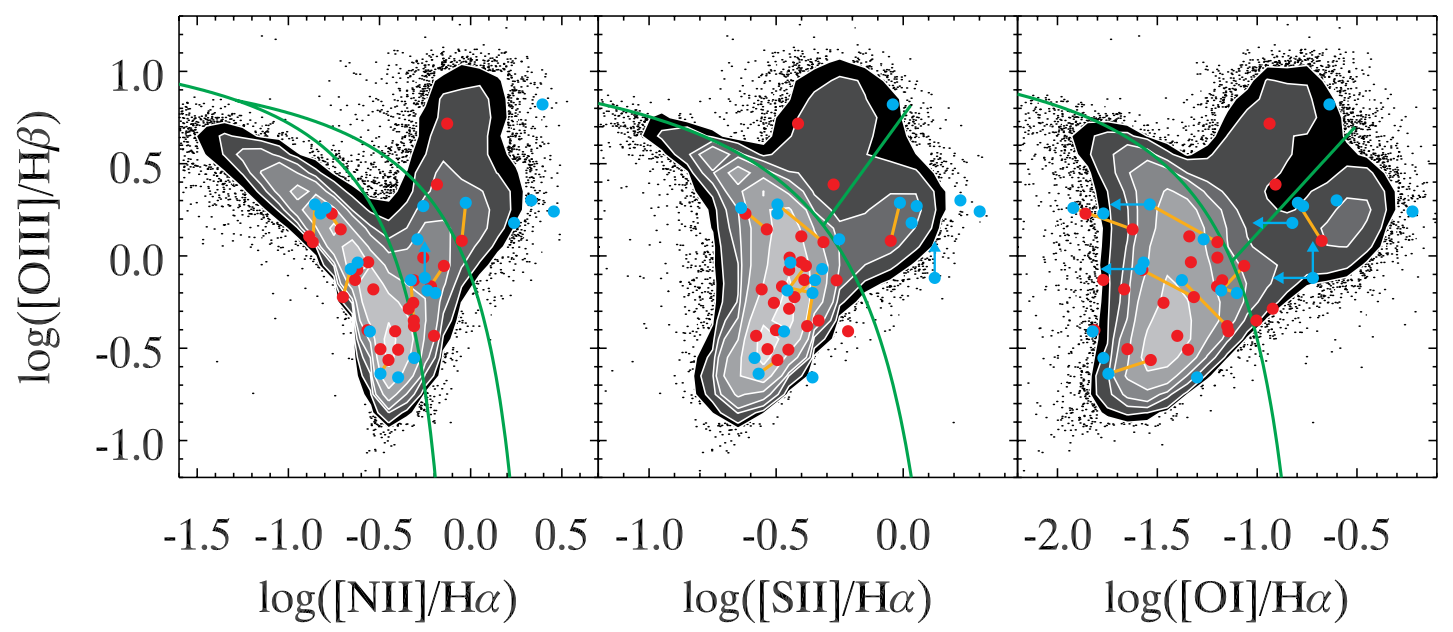

FIG. 3.-Emission-line diagrams for galaxies in our sample. Red filled circles show line ratios derived from SDSS DR6 data, while blue filled circles are from HFS97; orange lines connect galaxies with both types of spectra. Gray scale and small black points indicate the Garching reduction of the full SDSS DR4 sample. Green lines indicate the classification system adopted from Kewley et al. (2006).

data $(\S 3.2)$. We then examine the strength of the evidence for these detections being MBHs in $\S 3.3$.

\subsection{Emission-Line Spectroscopy}

Of the 176 galaxies in our sample, 70 had possible nuclear spectra available in the SDSS Data Release 6 (DR6; AdelmanMcCarthy et al. 2007). After visually inspecting the location of each spectrum, we found that 62 spectra were coincident with the galaxy nuclei. Emission-line fluxes determined after modeling of the underlying stellar populations were kindly provided by $\mathrm{C}$. Tremonti (2007, private communication) using the method described in Tremonti et al. (2004) and available for SDSS Data Release 4 (DR4) data. ${ }^{5}$ The DR6 spectra used differ somewhat from earlier spectra. Most notably, the spectrophotometric zero point has changed, increasing fluxes by $\sim 35 \%$ but leaving line ratios unchanged. In general, the line ratios from the DR6 spectra agree very well with those in DR4. The formal errors on the line fluxes were scaled by factors of 1.4-2.5 (depending on the line) to include errors in the continuum subtraction and flux calibration derived from sources with multiple spectra as described on the Garching DR4 Web site. ${ }^{6}$ These errors were then propagated to the line ratios. We select emission-line galaxies in the SDSS spectrum by requiring that three of four strong lines $(\mathrm{H} \beta$, [O III] $\lambda 5007, \mathrm{H} \alpha$, and $\left[\mathrm{N}_{\mathrm{II}}\right]$ 26584) have detections above $3 \sigma$. Of the 62 spectra in the sample, 25 meet this criterion.

We also find 23 galaxies that were observed as part of the Palomar Survey (Filippenko \& Sargent 1985), 20 of which have detected emission lines (Ho et al. 1997b, hereafter HFS977 ). As with the SDSS data used above, the emission-line measurements are made after subtraction of the underlying stellar population based on spectral modeling. Errors in the line ratios were estimated using the data quality flags and assuming a conservative baseline uncertainty of $30 \%$, with $50 \%$ and $100 \%$ uncertainties for sources with uncertainty flags of " $b$ " and "c," respectively. Ten of these galaxies overlap with the SDSS spectra, giving a total of 75 galaxies for which we have nuclear spectra.

Of the 75 galaxies for which we have nuclear spectra, 39 have weak or undetected emission lines; we classify these galaxies

\footnotetext{
5 Available at http://www.mpa-garching.mpg.de/SDSS/DR4/.

6 See http://www.mpa-garching.mpg.de/SDSS/DR4/raw_data.html.

We use the emission-line measurements from HFS97, but not their spectral classifications.
}

as having absorption-dominated spectra. For the remaining 36 emission-line spectra, we followed the classification scheme of Kewley et al. (2006) to separate the sample into star-forming galaxies, composite objects, or Seyfert and LINER AGNs. This classification scheme relies on four line ratios, $\left[\mathrm{O}_{\mathrm{III}}\right] / \mathrm{H} \beta,\left[\mathrm{O}_{\mathrm{I}}\right] / \mathrm{H} \alpha$, $[\mathrm{N} \mathrm{II}] / \mathrm{H} \alpha$, and $[\mathrm{S}$ II $] / \mathrm{H} \alpha$.

Three emission-line ratio diagrams (BPT diagrams; Baldwin et al. 1981) are shown in Figure 3. This figure shows line ratios from the full DR4 sample of galaxies in gray-scale contours and small black points along with our sample galaxies as red (SDSS) and blue (HFS97) filled circles. The green lines indicate the demarcation lines used in the classification scheme (Kewley et al. 2006). The primary classification of galaxies into star-forming, composite objects, or AGNs is done using the $[\mathrm{N}$ II] $/ \mathrm{H} \alpha$ versus $[\mathrm{O}$ III $] / \mathrm{H} \beta$ diagram (Fig. 3, left panel) ${ }^{8}$ Spectra are classified as being AGNs if they fall in the upper right part of the diagram above the theoretical maximum starburst line of Kewley et al. (2001) in the $[\mathrm{N} \mathrm{II}] / \mathrm{H} \alpha$ versus $[\mathrm{O}$ III $] / \mathrm{H} \beta$ diagram. Composite objects have emission lines thought to be caused by a mix of AGNs and star-forming lines and have line ratios falling below the Kewley et al. (2001) line and above the empirical Kauffmann et al. (2003) line. Galaxies below the Kauffmann et al. (2003) line have line ratios dominated by star formation. Further separation of AGNs into LINER and Seyfert galaxies is done using the $\left[\mathrm{S}_{\mathrm{II}}\right] / \mathrm{H} \alpha$ versus $\left[\mathrm{O}_{\mathrm{III}}\right] / \mathrm{H} \beta$ and $\left[\mathrm{O}_{\mathrm{I}}\right] / \mathrm{H} \alpha$ versus $\left[\mathrm{O}_{\mathrm{III}}\right] / \mathrm{H} \beta$ diagrams (Fig. 3 , middle and right panels), with Seyfert galaxies lying at higher values of $[\mathrm{O} \mathrm{III}] / \mathrm{H} \beta$.

Using the $[\mathrm{N} \mathrm{II}] / \mathrm{H} \alpha$ versus $[\mathrm{O}$ III] $/ \mathrm{H} \beta$ diagram, we classify 18 galaxies as having star-forming ("H II") spectra, 11 as having composite spectra ("C"), and seven as having AGN-like spectra ("AGN"). The other line ratio diagrams suggest that three of the AGNs are Seyfert galaxies ("S2") and four are LINERs ("L2"), while an additional three composite galaxies are also found to have LINER-like line ratios. For the 10 overlapping galaxies (connected by orange lines in Fig. 3), the classifications between the HFS97 and SDSS data agree in all cases, except for NGC 5879. This galaxy is classified as an AGN from both spectra but is found to be a LINER only from the SDSS spectrum.

\footnotetext{
${ }^{8}$ Due to the proximity of the emission lines used in these ratios, the $[\mathrm{N} \mathrm{II} / \mathrm{H} \alpha$ vs. $[\mathrm{O} \mathrm{III}] / \mathrm{H} \beta$ diagram is remarkably insensitive to reddening: $5 \mathrm{mag}$ of reddening creates a change of 0.006 and $0.08 \mathrm{dex}$ in the $[\mathrm{N} \mathrm{II]} / \mathrm{H} \alpha$ and $[\mathrm{O} \mathrm{III]} / \mathrm{H} \beta$ ratios, respectively.
} 
TABLE 2

Optical Spectroscopy for Star-forming Galaxies

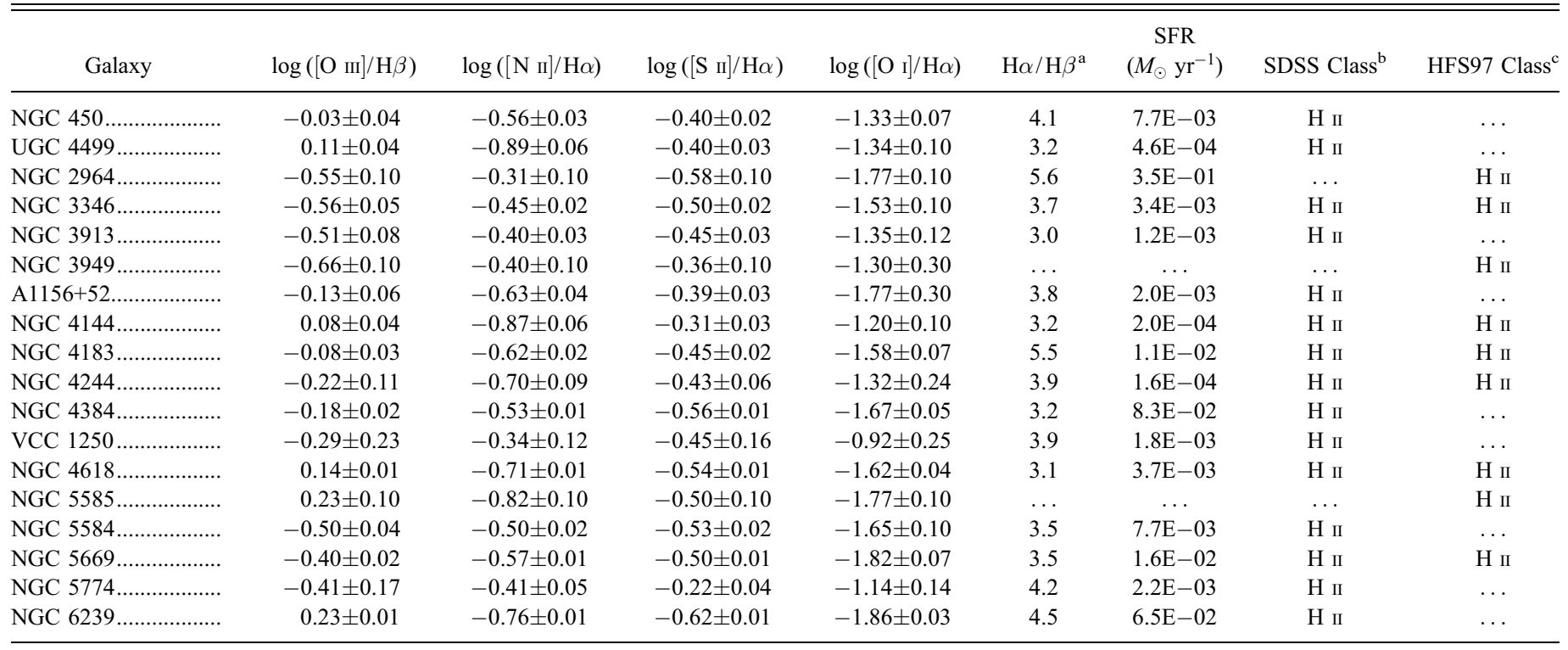

${ }^{\text {a }}$ Galaxies with no data for their $\mathrm{H} \alpha / \mathrm{H} \beta$ ratio and SFR are those for which the HFS97 data were not photometric.

b The classification of $\mathrm{H}$ II indicates a star-forming spectra $(\S 3.1)$.

${ }^{c}$ This column gives our classification of the HFS97 line ratios.

Our classifications also agree well with the somewhat different classification system used by HFS97 for the Palomar galaxies. ${ }^{9}$ Eleven of the 18 sources we classified as composite or AGN are in the HFS97 sample. Of these, 8 are classified as transition or AGN by HFS97. Specifically, the five galaxies that we classify as LINER and Seyfert AGN match their classifications exactly, while for the six galaxies we classified as composite, half of them were classified as star-forming, with the other half being LINERs or transition objects in their classification scheme. Both the composite class from Kewley et al. (2006) and the transition class from HFS97 are thought to result from a mix of star-forming and AGN spectra; thus, a correspondence between these classes is expected. In the HFS97 classification the transition objects are objects with a mix of LINER and star-forming spectra, while in our classification they can be a mix of LINER or Seyfert and star-forming spectra.

One of the galaxies in the HFS97 sample, NGC 4750, was found to have broad $\mathrm{H} \alpha$ emission and is thus classified by them as L1.9. We have adopted this classification. Also, Shields et al. (2008) have recently found that the [N II] line in the composite galaxy NGC 1042 has high-velocity $\left(300 \mathrm{~km} \mathrm{~s}^{-1}\right)$ wings. Visual inspection of the model-subtracted SDSS spectra suggests that none have any obvious broad emission lines.

The line ratios used in our classification are given in Table 2 for the star-forming galaxies and in Table 3 for the composite and AGN galaxies (C/AGN). For the star-forming nuclei, Table 2 also gives the star formation rates based on $\mathrm{H} \alpha$ luminosities derived from the relation given in Kennicutt (1998). Table 3 gives the [O III] luminosities for the C/AGN nuclei that are used in $\S 5.2$. These line luminosities have been corrected for reddening assuming $\mathrm{H} \alpha / \mathrm{H} \beta=2.85$ for the star-forming galaxies and $\mathrm{H} \alpha / \mathrm{H} \beta=$ 3.1 for the C/AGN galaxies (Osterbrock 1989; Kewley et al. 2006).

To get a sense of the uncertainty in our classification, we repeated the classification after both adding and subtracting the

\footnotetext{
9 The HFS97 classification system gives a primary role to the [O $\mathrm{I}] / \mathrm{H} \alpha$ line ratio, which is quite weak in many of our spectra and significantly more susceptible to reddening than the $[\mathrm{N} \mathrm{II]} / \mathrm{H} \alpha$ ratio.
}

$1 \sigma$ errors to the $[\mathrm{N}$ II $] / \mathrm{H} \alpha$ and $[\mathrm{O}$ III $] / \mathrm{H} \beta$ ratios. This changes the classification for a handful of galaxies: the number of composite galaxies varies between 8 and 12, while the number of AGNs varies between 6 and 8. Seven galaxies have error bars crossing the $\mathrm{H}$ iI/C boundary: C-classified NGC 428, NGC 3423, NGC 4206, NGC 4517, and NGC 4625 and H II-classified NGC 2964 and VCC 1250. Two galaxies have error bars crossing the AGN/C boundary: C-classified VCC 1619 and AGN-classified NGC 4411B.

Although the galaxies in our sample are quite nearby, the physical resolution of the spectra ( $3^{\prime \prime}$ fibers for SDSS, $2^{\prime \prime}$ slit for HFS97) is still significantly larger than the typical cluster sizes. For a galaxy at the median distance of our sample (16.5 Mpc), the corresponding spatial resolution is $\sim 200 \mathrm{pc}$. This could lead to detection of star formation not coincident with the nucleus (Shields et al. 2007) and might be expected to dilute weak AGN emission, therefore causing genuine AGNs to be classified as composite objects.

In summary, from the 75 galaxies with available optical spectra, we find 18 that have composite or AGN spectra. We discuss the fraction of these galaxies that have MBHs in $\S 3.3$. We now discuss the radio and X-ray properties of our sample galaxies.

\subsection{Radio and X-Ray Correlations}

In order to explore the multiwavelength properties of the galaxies in our sample, we matched the NC positions to a number of radio and $\mathrm{X}$-ray catalogs. While the cataloged observations at these wavelengths are unable to resolve structures on the subarcsecond scale of the NCs, detections in the radio and/or the $\mathrm{X}$-ray regimes can be used to place limits on the emission from putative AGNs independently of the information derived from optical spectra.

\subsubsection{Radio Data}

Galaxies that are radio sources are thought to host either active star formation or AGNs. Radio observation of AGNs shows that they have compact nuclear radio sources with high brightness 
TABLE 3

Optical Spectroscopy for Composite and AGN Galaxies

\begin{tabular}{|c|c|c|c|c|c|c|c|c|}
\hline Galaxy & $\log ([\mathrm{O} \mathrm{III}] / \mathrm{H} \beta)$ & $\log ([\mathrm{N} \mathrm{II]} / \mathrm{H} \alpha)$ & $\log \left(\left[\mathrm{S}_{\mathrm{II}}\right] / \mathrm{H} \alpha\right)$ & $\log \left(\left[\mathrm{O}_{\mathrm{I}}\right] / \mathrm{H} \alpha\right)$ & $\mathrm{H} \alpha / \mathrm{H} \beta^{\mathrm{a}}$ & $L_{[\mathrm{O} \text { ㅍ }]} / L_{\odot}$ & SDSS Class $^{\mathrm{b}}$ & HFS97 Class ${ }^{\mathrm{c}}$ \\
\hline NGC 428........................... & $-0.12 \pm 0.10$ & $-0.25 \pm 0.10$ & $0.12 \pm 0.10$ & $<-0.72$ & $\ldots$ & & $\ldots$ & $\mathrm{C} / \mathrm{L} 2$ \\
\hline 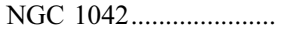 & $-0.01 \pm 0.02$ & $-0.26 \pm 0.01$ & $-0.45 \pm 0.02$ & $-1.20 \pm 0.04$ & 3.6 & $6.7 \mathrm{E}+04$ & $\mathrm{C}$ & $\ldots$ \\
\hline 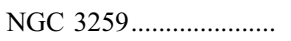 & $0.72 \pm 0.04$ & $-0.13 \pm 0.03$ & $-0.41 \pm 0.04$ & $-0.94 \pm 0.06$ & 4.9 & $5.6 \mathrm{E}+05$ & $\mathrm{AGN} / \mathrm{S} 2$ & . \\
\hline 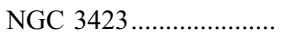 & $-0.38 \pm 0.10$ & $-0.31 \pm 0.05$ & $-0.38 \pm 0.05$ & $-1.15 \pm 0.14$ & 3.4 & $3.4 \mathrm{E}+03$ & $\mathrm{C}$ & $\mathrm{C}$ \\
\hline 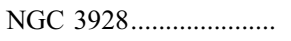 & $-0.25 \pm 0.02$ & $-0.31 \pm 0.01$ & $-0.51 \pm 0.01$ & $-1.47 \pm 0.04$ & 3.2 & $1.3 \mathrm{E}+05$ & $\mathrm{C}$ & $\ldots$ \\
\hline NGC 4517 ........................ & $0.09 \pm 0.17$ & $-0.29 \pm 0.10$ & $-0.25 \pm 0.10$ & $-1.27 \pm 0.10$ & 7.7 & $5.2 \mathrm{E}+04$ & $\ldots$ & $\mathrm{C} / \mathrm{L} 2$ \\
\hline 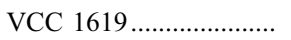 & $0.27 \pm 0.10$ & $-0.26 \pm 0.10$ & $0.05 \pm 0.10$ & $-0.77 \pm 0.10$ & $\ldots$ & & $\ldots$ & $\mathrm{C} / \mathrm{L} 2$ \\
\hline 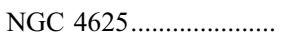 & $-0.35 \pm 0.10$ & $-0.31 \pm 0.05$ & $-0.33 \pm 0.05$ & $-1.00 \pm 0.10$ & 3.2 & $2.1 \mathrm{E}+03$ & $\mathrm{C}$ & $\ldots$ \\
\hline 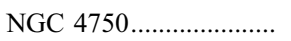 & $0.24 \pm 0.10$ & $0.46 \pm 0.10$ & $0.30 \pm 0.10$ & $-0.22 \pm 0.10$ & 3.3 & $2.0 \mathrm{E}+05$ & $\ldots$ & AGN/L1.9 \\
\hline 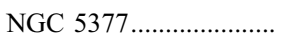 & $0.30 \pm 0.17$ & $0.33 \pm 0.10$ & $0.22 \pm 0.10$ & $-0.60 \pm 0.10$ & 4.6 & $5.8 \mathrm{E}+05$ & $\ldots$ & $\mathrm{AGN} / \mathrm{L} 2$ \\
\hline 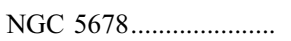 & $-0.17 \pm 0.08$ & $-0.22 \pm 0.03$ & $-0.48 \pm 0.05$ & $-1.20 \pm 0.12$ & 7.0 & $3.7 \mathrm{E}+05$ & $\mathrm{C}$ & $\mathrm{C}$ \\
\hline 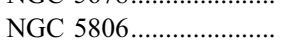 & $-0.05 \pm 0.07$ & $-0.15 \pm 0.05$ & $-0.38 \pm 0.06$ & $-1.07 \pm 0.14$ & 4.1 & $8.4 \mathrm{E}+04$ & $\mathrm{C}$ & $\mathrm{C}$ \\
\hline
\end{tabular}

${ }^{\text {a }}$ Galaxies with no data for their $\mathrm{H} \alpha / \mathrm{H} \beta$ ratio and [O III] luminosity are those for which the HFS97 data were not photometric.

b Classes are defined in $\S 3.1$; "C" = composite, "L" = LINER, and "S" = Seyfert.

c This column gives our classification of the HFS97 line ratios.

temperatures that cannot be reproduced by starbursts (e.g., Terashima \& Wilson 2003). Such radio sources are detected in about one-third of galaxies classified as composite or AGN objects in the Palomar survey (Nagar et al. 2005). Sources with $L_{1.4 \mathrm{GHz}} \geq 10^{23} \mathrm{~W} \mathrm{~Hz}^{-1}$ are generally called radio-loud AGNs and are unambiguous evidence of an AGN (Best 2004). However, these sources are typically associated with very massive galaxies; thus, it is unlikely that we will find radio-loud AGNs in our sample (Croft et al. 2007).

We used the Very Large Array (VLA) Faint Images of the Radio Sky at Twenty cm (FIRST; Becker et al. 1995) to search for radio counterparts to the galaxies in our sample. FIRST is the deepest large-scale radio survey currently available; the limiting flux density is about $1.0 \mathrm{mJy}$, the survey resolution is $\sim 5^{\prime \prime}$, and the footprint is roughly the same as that of SDSS. We queried FIRST for radio sources within $30^{\prime \prime}$ of our NCs and found
13 matches. ${ }^{10}$ The median separation between the radio source and the cluster position is $3^{\prime \prime} \pm 9^{\prime \prime}$ (see Table 4). Of the 13 matched galaxies, we have optical-based classifications for 9 (see Table 4). One galaxy, NGC 5377, is classified as an AGN/L2, while three others are classified as composite objects in our analysis. The remaining five galaxies have star-forming optical spectra. Unsurprisingly, the $L_{1.4 \mathrm{GHz}}$ for all these objects is well below $10^{23} \mathrm{~W} \mathrm{~Hz}^{-1}$.

Visual inspection of the corresponding radio images did not find morphological evidence for the presence of an AGN (e.g.,

${ }^{10}$ Matching to the National Radio Astronomy Observatory (NRAO) VLA Sky Survey (NVSS; Condon et al. 1998), which covers the sky north of $\delta=-40^{\circ}$ and includes sources stronger than $\sim 2.5 \mathrm{mJy}$, returned a larger number of matches, 52 , including all of the FIRST-detected objects. However, the resolution for NVSS is roughly $45^{\prime \prime}$ FWHM and the survey data are therefore far less useful for our purposes.

TABLE 4

Radio Data for the 13 Galaxies with FiRST Matches

\begin{tabular}{|c|c|c|c|c|c|c|c|}
\hline Galaxy & Optical Class & $\begin{array}{c}\text { Separation NC } \\
\quad(\operatorname{arcsec})\end{array}$ & $\begin{array}{l}\text { Integrated Flux } \\
(\mathrm{mJy})\end{array}$ & $\begin{array}{c}\mathrm{rms} \\
(\operatorname{arcsec})\end{array}$ & $\begin{array}{l}\text { Major Axis } \\
\quad(\operatorname{arcsec})\end{array}$ & $\begin{array}{l}\text { Minor Axis } \\
(\operatorname{arcsec})\end{array}$ & $\begin{array}{c}L_{1.4 \mathrm{GHz}} \\
\left(\mathrm{W} \mathrm{Hz} \mathbf{H z}^{-1}\right)\end{array}$ \\
\hline NGC $2964 \ldots \ldots \ldots \ldots \ldots . .$. & $\mathrm{H}$ II & 0.2 & 28.38 & 0.14 & 6.98 & 4.99 & $1.5 \times 10^{21}$ \\
\hline NGC $3177 \ldots \ldots \ldots \ldots$ & $\mathrm{C}$ & 1.0 & 26.21 & 0.24 & 9.47 & 7.56 & $1.3 \times 10^{21}$ \\
\hline 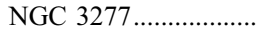 & $\ldots$ & 0.6 & 3.29 & 0.14 & 7.40 & 6.77 & $1.9 \times 10^{20}$ \\
\hline 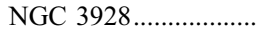 & $\mathrm{C}$ & 0.7 & 8.55 & 0.14 & 10.11 & 8.99 & $2.6 \times 10^{20}$ \\
\hline 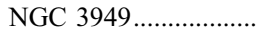 & $\mathrm{H}$ II & 24.9 & 21.08 & 0.14 & 23.21 & 17.68 & $5.4 \times 10^{20}$ \\
\hline NGC $4030 \ldots \ldots \ldots \ldots$ & $\ldots$ & 10.0 & 34.26 & 0.16 & 41.07 & 26.28 & $1.8 \times 10^{21}$ \\
\hline NGC $4144 \ldots \ldots \ldots \ldots \ldots$ & $\mathrm{H}$ II & 14.9 & 1.56 & 0.14 & 3.82 & 0.00 & $9.8 \times 10^{18}$ \\
\hline VCC 437 ......................... & $\ldots$ & 25.9 & 4.94 & 0.13 & 9.19 & 8.38 & $1.6 \times 10^{20}$ \\
\hline 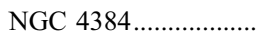 & $\mathrm{H}$ II & 3.3 & 12.52 & 0.15 & 14.39 & 10.39 & $2.3 \times 10^{21}$ \\
\hline NGC $4701 \ldots \ldots \ldots \ldots . . . .$. & $\ldots$ & 3.6 & 5.93 & 0.15 & 18.66 & 8.88 & $8.8 \times 10^{19}$ \\
\hline 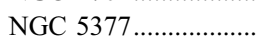 & $\mathrm{AGN} / \mathrm{L} 2$ & 1.4 & 3.26 & 0.14 & 6.94 & 6.61 & $3.8 \times 10^{20}$ \\
\hline NGC $5678^{\mathrm{a}} \ldots \ldots \ldots \ldots$ & C & 7.2 & 68.39 & 0.14 & 44.03 & 32.26 & $6.6 \times 10^{21}$ \\
\hline 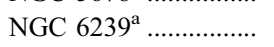 & $\mathrm{H}$ II & 0.8 & 4.11 & 0.17 & 11.96 & 5.43 & $1.4 \times 10^{20}$ \\
\hline
\end{tabular}

Note.-All of these galaxies were also detected by NVSS.

${ }^{\text {a }}$ Flagged as a possible sidelobe of a nearby bright source. NGC 5377 has two other fainter FIRST sources within $20^{\prime \prime}$ of the NC position; NGC 6239 has no other FIRST sources within $60^{\prime \prime}$. It is unclear why these radio sources are flagged. 


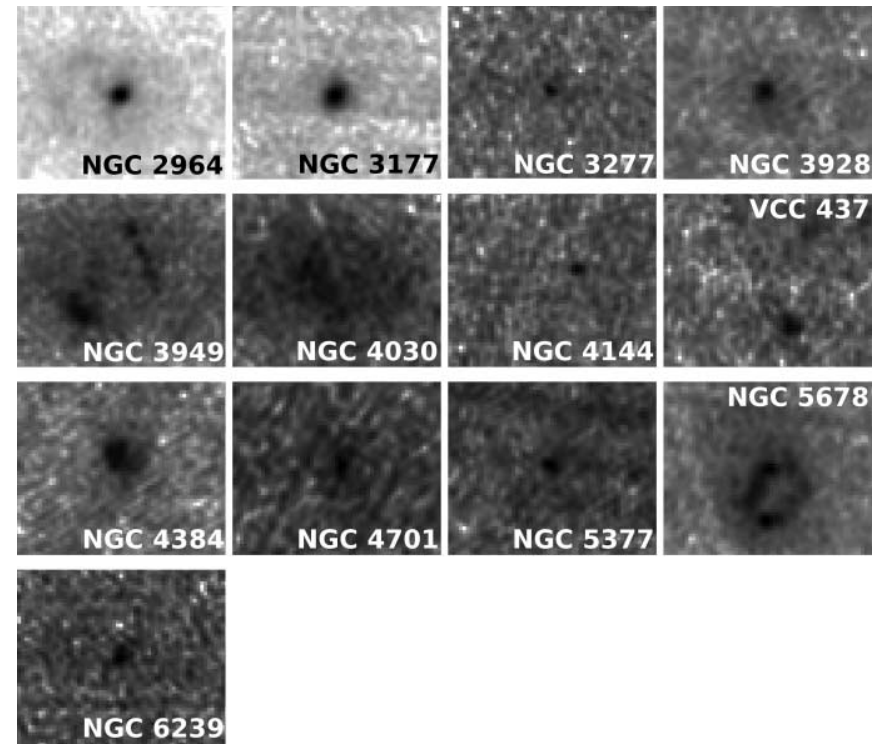

FIG. 4.-FIRST images of the 13 galaxies for which we find a catalog match within $30^{\prime \prime}$. Each image is roughly $1.6^{\prime}$ in height and $2^{\prime}$ in width and centered on the optical position of the $\mathrm{NC}$ in the galaxy.

jets) in any of these (see Fig. 4). In most cases, the radio emission appears diffuse, as expected if it is due to star formation. However, NGC 5377 and two of the three composite objects (NGC 3177 and NGC 3928) with radio detections appear as point sources in the FIRST images, consistent with the presence of a possible nuclear AGN source. For NGC 5377, higher resolution observations do confirm this source as an AGN (Nagar et al. 2005); similar radio observations are needed to determine the nature of the other sources.

An additional 94 galaxies from our sample fall within the FIRST footprint but do not have detections. White et al. (2007) have developed a method for obtaining the FIRST radio flux density for a group of sources when individual group members are undetected in the survey. Accordingly, we calculated a stacked image for our undetected sources. We applied the correction, prescribed in White et al. (2007), to account for the snapshot bias and derived our errors from the bootstrapping method (Efron 1982), which tests how individual entries affect the stacked average. We found that the average radio flux density for the undetected galaxies in the FIRST footprint is $\sim 90 \pm 20 \mu \mathrm{Jy}$ (whereas the detected galaxies have flux densities of 1.6-68 mJy). This stacked "detection" provides an average measurement of the radio intensity for the typical nuclear region in these galaxies. At best, this suggests that any putative radio AGN in these galaxies is extremely weak. We note that among these nondetections are four galaxies we have classified as C/AGN objects using optical spectroscopy: NGC 4411B, VCC 1619, NGC 5806, and NGC 5879.

\subsubsection{X-Ray Data}

$\mathrm{X}$-rays are one of the most direct evidences of nuclear activity, and X-ray observations are therefore essential in revealing the accretion processes taking place near the central black holes in AGNs. With the spatial resolution of the new generation of $\mathrm{X}$-ray telescopes, it is becoming possible to isolate low-luminosity AGNs from other X-ray sources in a galaxy (e.g., Ho et al. 2001). In particular, the ability to detect photons with energies of several $\mathrm{keV}$ allows for the uncovering of AGNs hidden at other wavelengths by column densities as high as $N_{\mathrm{H}} \sim 10^{24} \mathrm{~cm}^{-2}$ (Ho et al. 2001).
We matched our sample to the combined ROSAT All Sky Survey (RASS) Bright and Faint Source Catalogs (Voges et al. 1999). ${ }^{11}$ We also queried the High Energy Astrophysics Science Archive Research Center Web site for matches from the various catalogs of pointed ROSAT observations, Chandra sources, and XMM-Newton detections. ${ }^{12}$

The X-ray data obtained in this fashion are rather heterogeneous, since the queried catalogs and instruments are very different in nature. For example, while none of the Chandra or XMMNewton surveys cover as much sky as the RASS, the latter's positional accuracy is generally relatively poor (typically at least $15^{\prime \prime}$ ). In a similar vein, Chandra and XMM-Newton both have greater sensitivity than ROSAT, but the telescopes are not designed to detect sources in exactly the same energy ranges.

In order to produce the data for the matches listed in Table 5, we proceeded as follows:

1. Since Chandra typically has better positional accuracy than $X M M-N e w t o n$, and both typically have better accuracy than ROSAT, we used Chandra data preferentially, then XMM-Newton, then ROSAT, in our analysis.

2. For galaxies with Chandra and/or XMM-Newton detections, there were frequently multiple X-ray sources. In those cases we chose the source closest to the optical position of the NC. ${ }^{13}$ The median offset for the 13 Chandra/XMM-Newton sources is $1.3^{\prime \prime} \pm 1.4^{\prime \prime}$, with the largest offset being $4.9^{\prime \prime}$. All the Chandra data come from XAssist (an automated extraction pipeline for X-ray data; Ptak \& Griffiths 2003); these are the sources with an " $\mathrm{X}$ " prefix in Table 5. The XMM-Newton data are from the second catalog of the XMM-Newton serendipitous survey (2XMM sources; M. Watson et al. 2008, in preparation). ${ }^{14}$

3 . Of the eight galaxies with RASS matches, half had detections in pointed catalogs, and we therefore used those data to characterize the X-ray sources. Three of the other galaxies, NGC 3259, NGC 4030, and NGC 4540, are published X-ray sources (identified as such by Véron-Cetty et al. 2004; Moran et al. 1996; Mickaelian et al. 2006). The positional offsets between the RASS source and the NC are small $\left(\leq 10^{\prime \prime}\right)$ for the first two galaxies, and the associations with the RASS sources seem secure. However, NGC 4540 and NGC 2566 both have large positional uncertainties and offsets from the NCs. Pointed observations are clearly required to confirm that these two galaxies are X-ray sources, and we consider these associations to be tentative.

4. Four more galaxies are included in various ROSAT pointed surveys and/or identified as X-ray sources in the literature. Two of these are from catalogs of High Resolution Imager (HRI) sources (Panzera et al. 2003; PSPC catalog ${ }^{15}$ ). ${ }^{16}$ In both these galaxies, NGC 1385 and NGC 6000, the offset with the NC is less than the $5^{\prime \prime}$ nominal positional accuracy for HRI observations (Flesch \& Hardcastle 2004). The two other sources were detected with the other X-ray instrument aboard ROSAT, the Position Sensitive Proportional Counter (PSPC), whose positional

11 Vizier Online Data Catalog, 9029 (W. Voges et al. 2000).

12 This research has made use of data obtained from the High Energy Astrophysics Science Archive Research Center (HEASARC), provided by NASA's Goddard Space Flight Center.

${ }^{13}$ The positional accuracy of our NC positions is dominated by the uncertainty in the HST astrometry, which is typically accurate to within $1^{\prime \prime}-2^{\prime \prime}$.

14 The catalog is available at http://xmm.vilspa.esa.es/xsa/.

15 Vizier Online Data Catalog, 9028 (ROSAT Scientific Team, 2000).

16 The Panzera et al. (2003) catalog is a reanalysis of the HRI observations with exposures longer than $100 \mathrm{~s}$; the other catalog includes all HRI pointed observations. 
TABLE 5

X-Ray Data for the 22 GalaXies with Chandra, ROSAT, or XMM-Newton Matches

\begin{tabular}{|c|c|c|c|c|c|}
\hline Galaxy & Optical Class $^{\mathrm{a}}$ & X-Ray Source Name & $\begin{array}{c}\text { Separation NC } \\
\quad(\operatorname{arcsec})\end{array}$ & $\begin{array}{c}\text { Extent } \\
(\operatorname{arcsec})\end{array}$ & $\begin{array}{c}L_{\mathrm{X}}(2-10 \mathrm{keV})^{\mathrm{b}} \\
\quad\left(\operatorname{ergs~s}^{-1}\right)\end{array}$ \\
\hline \multicolumn{6}{|c|}{ Chandra } \\
\hline VCC 751 & $\ldots$ & $\mathrm{X} 122448.46+181141.8$ & 1.3 & $2.0 \times 2.0$ & $1.58 \times 10^{38}$ \\
\hline 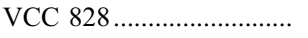 & ABS & $\mathrm{X} 122541.63+124837.3$ & 1.3 & $5.0 \times 5.0$ & $3.25 \times 10^{38}$ \\
\hline VCC $1192 \ldots \ldots \ldots \ldots \ldots . .$. & $\ldots$ & $\mathrm{X} 122930.21+075934.6$ & 0.8 & $0.7 \times 0.6$ & $2.37 \times 10^{38}$ \\
\hline  & AGN/L1.9 & $\mathrm{X} 125007.34+725228.8$ & 1.0 & $0.4 \times 0.4$ & $1.05 \times 10^{40}$ \\
\hline 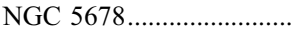 & $\mathrm{C}$ & X143205.54+575517.2 & 0.5 & $0.7 \times 0.3$ & $6.67 \times 10^{38}$ \\
\hline 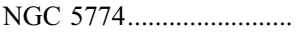 & $\mathrm{H}$ II & $\mathrm{X} 145342.77+033503.2$ & 4.9 & $0.7 \times 0.6$ & $1.17 \times 10^{38}$ \\
\hline NGC 5879 ............................ & $\mathrm{AGN} / \mathrm{L} 2$ & $\mathrm{X} 150946.72+570000.2$ & 1.0 & $1.0 \times 0.5$ & $2.51 \times 10^{38}$ \\
\hline \multicolumn{6}{|c|}{ ROSAT } \\
\hline 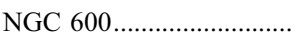 & $\ldots$ & 2RXP J013305.7-071835 & 9.8 & 0 & $8.10 \times 10^{39}$ \\
\hline NGC $1385^{\mathrm{c}} \ldots \ldots \ldots \ldots \ldots \ldots$ & $\ldots$ & 1BMW 033728.0-243003 & 4.1 & 14 & $1.13 \times 10^{40}$ \\
\hline 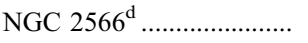 & $\ldots$ & 1RXS J081847.0-252922 & 40.7 & 0 & $2.69 \times 10^{40}$ \\
\hline 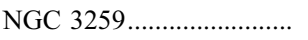 & AGN/S2 & RX J103234+65024 & 9.4 & 0 & $1.27 \times 10^{40}$ \\
\hline NGC $3445 \ldots \ldots \ldots \ldots \ldots . .$. & $\ldots$ & 1WGA J1054.5+5659 & 9.4 & $\ldots$ & $2.66 \times 10^{39}$ \\
\hline NGC $4030 \ldots \ldots \ldots \ldots \ldots \ldots \ldots . .$. & $\ldots$ & RX J120023-01055 & 10.8 & 9 & $1.47 \times 10^{40}$ \\
\hline 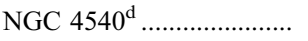 & $\ldots$ & 1RXS J123456.0+153314 & 74.1 & 0 & $7.60 \times 10^{40}$ \\
\hline NGC $6000^{\mathrm{c}} \ldots \ldots \ldots \ldots \ldots \ldots$ & $\ldots$ & 1RXH J154949.7-292310 & 3.6 & 0 & $5.83 \times 10^{40}$ \\
\hline \multicolumn{6}{|c|}{ XMM-Newton } \\
\hline 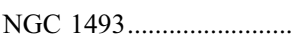 & $\ldots$ & 2XMM J035727.3-461239 & 4.5 & $<6$ & $3.51 \times 10^{38}$ \\
\hline 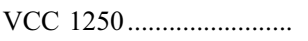 & $\mathrm{H}$ II & 2XMM J122959.1+122052 & 3.0 & $<6$ & $3.23 \times 10^{38}$ \\
\hline 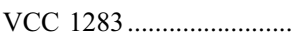 & ABS & 2XMM J123018.3+133440 & 0.7 & $<6$ & $2.82 \times 10^{38}$ \\
\hline VCC 1355 & $\ldots$ & 2XMM J123120.2+140656 & 1.8 & $<6$ & $4.37 \times 10^{38}$ \\
\hline 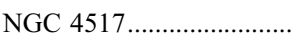 & $\mathrm{C}$ & 2XMM J123245.4+000655 & 1.8 & $<6$ & $7.25 \times 10^{38}$ \\
\hline 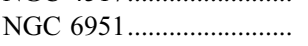 & AGN/S2 & 2 XMM J203714.0+660619 & 0.5 & $<6$ & $5.39 \times 10^{39}$ \\
\hline 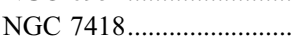 & $\ldots$ & 2 XMM J225636.0-370145 & 1.5 & $<6$ & $6.37 \times 10^{39}$ \\
\hline
\end{tabular}

a Classes identified in $\S 3.1$; “ABS" = absorption spectrum.

b Assuming $\Gamma=1.8$.

c ROSAT HRI detection.

d Tentatively associated with X-ray source.

accuracy is closer to $30^{\prime \prime}$. Flesch \& Hardcastle (2004) identify NGC 600 as a PSPC source with $61 \%$ confidence; ${ }^{17}$ the offset between the X-ray source (positional uncertainty $13^{\prime \prime}$ ) and the $\mathrm{NC}$ is $10^{\prime \prime}$. NGC 3445 is included in WGACAT. ${ }^{18}$

5. We used WebPIMMS (Mukai 1993) to calculate $2-10 \mathrm{keV}$ fluxes for all of these sources, assuming a canonical intrinsic powerlaw spectrum with photon index $\Gamma=1.8$ (for low-luminosity AGNs $\Gamma$ ranges between 1.6 and 2.0; Terashima \& Wilson 2003) absorbed to the Galactic value. We then calculate the X-ray luminosities given in the seventh column of Table 5. These values are consistent with those in the literature for the known X-rayemitting galaxies, once differences in the adopted distances and energy bands are taken into account.

A complicating factor in interpreting the X-ray luminosities we obtain at the end of this process is the differing point-spread function (PSF) for each of these telescopes. While, broadly speaking, Chandra, XMM-Newton, and the HRI on ROSAT have similar PSFs (from $\sim 1^{\prime \prime}$ to $6^{\prime \prime}$ ), the ROSAT PSPC has a PSF with $\mathrm{a} \sim 30^{\prime \prime}$ FWHM (Panzera et al. 2003), and the PSFs all vary with position on the detector and photon energy. Determining which sources are extended and which are truly pointlike is therefore difficult, with the exception of the Chandra sources, for which

\footnotetext{
${ }^{17}$ Flesch \& Hardcastle (2004) use the ROSAT Scientific Team catalog of PSPC pointed observations as the basis for this match.

${ }^{18}$ Vizier Online Data Catalog, 9012 (N. E. White et al. 1996). They generated their own point-source catalog from all publicly available ROSAT PSPC observations.
}

XAssist provides subarcsecond measurements of source extent (listed in the sixth column of Table 5). The situation with the 2XMM sources is relatively straightforward: any source that is smaller than the 6" PSF and hence unresolved is set in the catalog to have a $0^{\prime \prime}$ extent.

The RASS sources (PSPC detections) all have associated cataloged extent measurements and extent likelihoods (see Voges et al. 1999); however, these values should be treated with caution (e.g., for bright sources the deviations of the PSF from a Gaussian lead to incorrect extent measurements) and are considered reliable only if the extent measurement is $>10^{\prime \prime}$ and the likelihood is $>10$ (F. Haberl 2007, private communication; see also Haberl et al. 2000). For three of the RASS sources the measured extent and extent likelihood are both $0^{\prime \prime}$; for the fourth, RX J12002301055 (NGC 4030), the cataloged extent is $9^{\prime \prime}$, but the extent likelihood is 1 , implying that the source is actually unresolved.

For the sources with pointed ROSAT observational data, the definition of source extension varies from catalog to catalog. For the two sources detected by the HRI, 1BMW 033728.0-243003 and 1RXH J154949.7-292310 (counterparts to NGC 1385 and NGC 6000), the extents are given as $0^{\prime \prime}$. However, Panzera et al. (2003) give the actual source extent for NGC 1385 as 14" (with extent likelihood 0). The catalog entry for NGC 6000 indicates that it is not extended but does not give an associated measurement. As for the PSPC sources, 2RXP J013305.7-071835 (NGC 600) is fitted by a Gaussian with $\sigma=12^{\prime \prime}$ and is given an extent likelihood of 0 in the PSPC catalog (see footnote 14). 
Finally, the WGACAT is defined as a point-source catalog, and no measurements are included in the available data.

In summary, we find a total of $22 \mathrm{X}$-ray sources associated with NCs in our sample. We evaluate how many of these may possess black holes in the following section.

\subsection{Do These Sources Harbor Black Holes?}

We now consider how reliably we can infer the presence of $\mathrm{MBHs}$ in the galaxies for which our multiwavelength data indicate the presence of AGNs.

For the optical data, considerable work has been done to determine whether low-luminosity Seyfert galaxies, LINERs, and transition/composite objects do in fact represent $\mathrm{MBH}$ accretion. While Seyfert line ratios are a strong indicator of $\mathrm{MBH}$ accretion, it is not clear whether the same is true for all LINERs and transition/composite objects (see review by Ho 2004). Several recent studies of LINER galaxies suggest that a majority of them do in fact indicate the presence of an $\mathrm{MBH}$ based on highresolution radio observations (Nagar et al. 2005), X-ray emission (Dudik et al. 2005; González-Martín et al. 2006), and UV variability (Maoz et al. 2005). There are cases where LINER line ratios do seem to be produced by star formation. For instance, in the LINER galaxy M61 (NGC 4303), the recently formed stars provide more than enough ionizing flux to explain the observed $\mathrm{H} \alpha$ emission (Colina et al. 2002), suggesting that LINERs may sometimes just be caused by bursts of star formation. However, we note that in the context of our sample, a majority of the LINERs are found in early-type spiral galaxies, whose NCs are known to only rarely host significant young stellar populations (Rossa et al. 2006). The nature of composite or transition objects, proposed to be a mix of AGN emission and star formation, is even more difficult to determine (Ho 2004). From a radio survey of the HFS97 catalog, Nagar et al. (2005) find that $16 \%$ of transition objects have compact radio detections, compared to $\sim 45 \%$ for the Seyfert galaxies and LINERs.

The high-velocity emission line wings observed by Shields et al. (2008) in NGC 1042 (which we classify as a composite galaxy) provide strong evidence for an $\mathrm{MBH}$ in that galaxy. In summary, the presence of an AGN implies the presence of MBHs reliably in Seyfert galaxies and a majority of LINERs, but less reliably for sources with composite spectra.

For the radio data, the presence of AGNs can only be safely inferred in cases where the emission is above a certain threshold. Below that threshold (typically $\sim 10^{23} \mathrm{~W} \mathrm{~Hz}^{-1}$; Best 2004), either star formation or low-level AGNs can be responsible for the radio emission. The 13 galaxies with FIRST detections in our sample all have luminosities $<10^{22} \mathrm{~W} \mathrm{~Hz}^{-1}$, which is unsurprising given the low masses of galaxies in our sample and the observed correlation between galaxy mass and radio-loudness (Croft et al. 2007). We therefore cannot use $L_{R}$ alone to identify radio AGNs in our sample. While morphological information could distinguish between star formation and AGN emission in galaxies with lower radio luminosities, the resolution of FIRST data is insufficient to place strong constraints on the nature of the sources in these galaxies. The radio data are therefore useful only in setting upper limits on any (low level) emission from MBHs in these galaxies, which require higher resolution observations to be detected with confidence.

For the X-ray data, which provide insight into a larger fraction of the AGN bolometric flux than the narrow optical emission lines, any well localized source with $L_{\mathrm{X}} \gtrsim 2 \times 10^{39} \mathrm{ergs} \mathrm{s}^{-1}$ very likely originates from an $\mathrm{MBH}$ (e.g., van der Marel 2004). This criterion is met by only four of our sources, NGC 4750 , NGC 6000, NGC 6951, and NGC 7418 (Table 5), two of which
(NGC 4750 and NGC 6951) have available optical spectra and are classified as AGNs. The other sources are either luminous but poorly resolved ROSAT PSPC detections or lower luminosity $\left(\sim 10^{38} \mathrm{ergs} \mathrm{s}^{-1}\right)$ Chandra or XMM-Newton detections. For the ROSAT PSPC detections, the resolution is quite poor (FWHM $\sim$ $\left.30^{\prime \prime}\right)$ and thus covers a significant portion of the galaxy $(\sim 3 \mathrm{kpc})$. The X-ray fluxes are within the range expected for normal galaxies (Shapley et al. 2001) and thus could originate from either collections of other X-ray sources or accretion onto an $\mathrm{MBH}$ (note that we include the extended ROSATHRI source in NGC 1385 in this category as well). For the higher resolution Chandra and $X M M-N e w t o n$ observations, which are localized to be coincident with the NC (see Table 5), the sources with low X-ray luminosities overlap with those expected for X-ray binaries (Fabbiano 2006). For instance, luminous X-ray sources in the NCs of M33 and NGC 2403 are both thought to be X-ray binaries (Dubus et al. 2004; Yukita et al. 2007). Also, low-mass X-ray binaries (LMXBs) are commonly found in massive clusters; in a survey of globular clusters in Virgo, $\sim 25 \%$ of the brightest globular clusters were found to have LMXBs (Sivakoff et al. 2007). The Chandra and $X M M-N e w t o n$ detections with $L_{\mathrm{X}} \lesssim 10^{39} \mathrm{ergs} \mathrm{s}^{-1}$ are therefore consistent with the presence of X-ray binaries in these NCs. However, many low-luminosity AGNs appear to have X-ray fluxes in this luminosity range as well (e.g., Dudik et al. 2005; Panessa et al. 2006). In conclusion, the presence of an MBH-powered source is strongly indicated for four X-ray sources, while for the remainder of the sources the X-ray energy could be resulting either from $\mathrm{MBH}$ accretion or from other X-ray sources.

Despite these uncertainties in identifying the presence of MBHs in single galaxies in our sample, the data presented here collectively provide good evidence that the overlap between NCs and MBHs is common. In total, 10 galaxies in our sample show strong evidence of an $\mathrm{MBH}$, with $\sim 30$ more having some indication of a possible $\mathrm{MBH}$. Furthermore, it is likely that many MBHs in our sample galaxies do not have enough activity to bring them to our attention; for example, neither the central black hole of the Milky Way (Narayan et al. 1998; Shields et al. 2007) nor that of M32 (Ho et al. 2003), galaxies similar to those in our sample, would likely be detected as AGNs in our study.

\section{PREVIOUSLY KNOWN AGN/NC SOURCES}

In this section we present galaxies previously known to host both an NC and an MBH or AGN. These can be added to our sample to explore the types of galaxies in which NCs and MBHs overlap. Furthermore, four galaxies presented below have measured black hole masses, enabling a comparison of their $\mathrm{NC}$ and $\mathrm{MBH}$ mass ( $§ 5.2$ ).

We find nine galaxies previously identified as having both $\mathrm{NCs}$ and an AGN or MBH, three of which are contained in our sample. These are all galaxies in which the presence of both an $\mathrm{NC}$ and $\mathrm{MBH} / \mathrm{AGN}$ is well substantiated (but see also Ghosh et al. 2006; Decarli et al. 2007).

The Milky Way hosts both an NC and an MBH. The NC in the Milky Way was first described by Becklin \& Neugebauer (1968) using IR observations and was found to have an FWHM of $3^{\prime}-5^{\prime}$ $(\sim 10 \mathrm{pc})$, consistent with NCs in similar galaxies (Carollo et al. 2002). More recent studies using both star counts and kinematics show that the mass enclosed within the central $10 \mathrm{pc}$ is $\sim 3 \times$ $10^{7} M_{\odot}$ (Genzel et al. 1996; Schödel et al. 2007). The Galactic $\mathrm{MBH}$ has a mass of $3.7 \times 10^{6} M_{\odot}$ (Ghez et al. 2005).

Another well-known galaxy with an $\mathrm{NC}, \mathrm{AGN}$, and $\mathrm{MBH}$ is NGC 4395, an Sm-type galaxy at a distance of 4.3 Mpc (Thim et al. 2004) with $M_{B}=-17.30$ (Paturel et al. 2003). The NC in NGC 4395 (Matthews et al. 1999) has an $M_{I}=-11.3$, an 

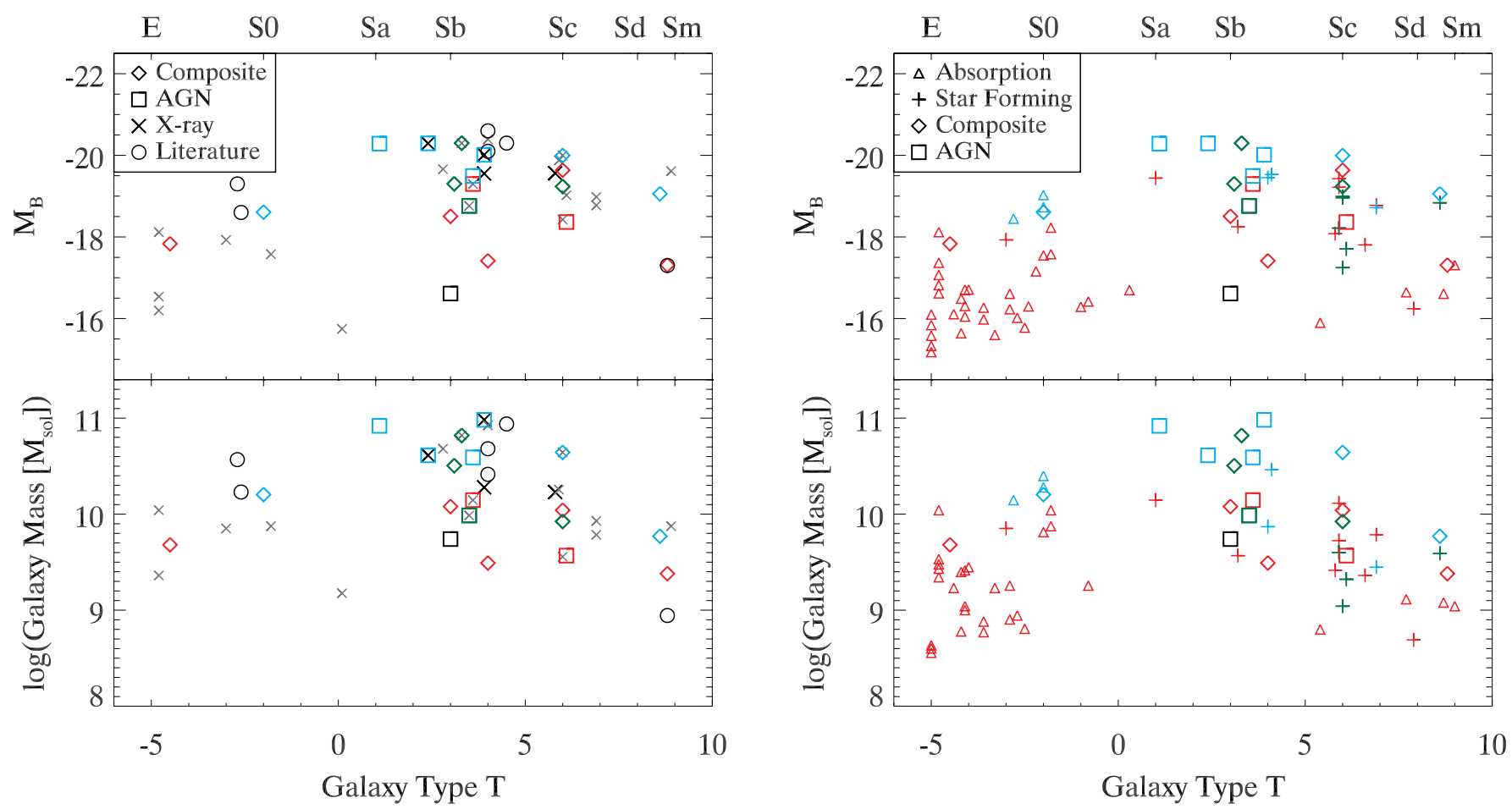

FIG. 5.-Left: Galaxy properties of all galaxies with NCs that also host candidate AGNs. The red symbols indicate spectra from HFS97, blue symbols are SDSS spectra, and green symbols have both HFS97 and SDSS spectra. The four X-ray sources that are very likely AGNs (see $\S 3.3$ ) are shown with large black crosses; the rest of the detections have smaller gray crosses. The six sources from the literature not contained within our sample are shown with circles (§ 4). The black square is ESO 205-G7 (see $\S 4$; Rossa et al. 2006). Right: Galaxy type and absolute magnitude of all galaxies with spectra, colored as in the left panels. Note that because mass estimates are not available for all the galaxies (see $\S 2$ ), some data points in the top panel are missing in the bottom panel.

effective radius of $0.19^{\prime \prime}(3.9 \mathrm{pc})$, and a velocity dispersion of $<30 \pm 5 \mathrm{~km} \mathrm{~s}^{-1}$ (Filippenko \& Ho 2003). Based on the velocity dispersion, Filippenko \& Ho (2003) suggest a mass for the NC of $\lesssim 6.2 \times 10^{6} M_{\odot}$. Using the method described in $\S 2.1$ to derive $\mathrm{NC}$ masses in late-type galaxies, we estimate the NC mass to be $1.1 \times 10^{6} M_{\odot}$. The AGN is one of the nearest and least luminous Seyfert 1 nuclei (Filippenko \& Sargent 1989). The black hole has a measured mass of $(3.6 \pm 1.1) \times 10^{5} M_{\odot}$ (Peterson et al. 2005), roughly one-third the mass of the NC.

Graham \& Driver (2007) note two elliptical/lenticular galaxies, NGC 3384 and NGC 7457, which have measured black hole masses (Tremaine et al. 2002) and apparent NCs. Although the nuclear sources in both cases are unresolved in HST observations presented by Ravindranath et al. (2001), both nuclear spectra are classified as absorption spectra by HFS97, suggesting that they are in fact NCs. Using distances from Jensen et al. (2003), we find the $M_{H}$ of the NCs of -15.44 and -15.71 for NGC 3384 and NGC 7457, respectively, corresponding to NC mass estimates of $2.1 \times 10^{7}$ and $2.7 \times 10^{7} M_{\odot}(\S 2.1)$.

Four sources in Scarlata et al. (2004) appear to have compact NCs and are classified as transition or AGN objects by HFS97. NGC 4321 (M100) and NGC 5921 are both Sbc galaxies with bright NCs $\left(M_{R} \sim-14\right)$ classified as transition objects in HFS97. NGC 4321 is undetected at X-ray and radio wavelengths despite targeted observations (Ho et al. 2001; Nagar et al. 2005). The two other galaxies (NGC 6384 and NGC 6951) are in our sample and are classified as LINER and Seyfert, respectively ( $\S 3.1$ ). Also in our sample is the Sb galaxy ESO 205-G7, mentioned in Rossa et al. (2006) as having a broad emission line spectrum based on unpublished VLT UVES spectra; however, this galaxy has no available spectrum or X-ray detection. Finally, the recent work by Gonzalez Delgado et al. (2008) analyzes HST WFPC2 archival data of AGNs in the HFS97 survey and includes obser- vations of numerous objects that may host both NCs and AGNs, including six of the galaxies for which we find overlap.

Possibly related to these galaxies are the recent detections of $\mathrm{MBHs}$ in the centers of two massive globular clusters thought to be the NCs of stripped dwarf galaxies (e.g., Meylan et al. 2001; Bedin et al. 2004). Dynamical observations of G1 and $\omega$ Cen suggest that they host black holes with masses $>10^{4} M_{\odot}($ Gebhardt et al. 2002, 2005; Noyola et al. 2006; Rasio et al. 2006). In G1, this claim has been further strengthened by detection of X-ray and radio emission consistent with a $2 \times 10^{4} M_{\odot}$ black hole (Pooley \& Rappaport 2006; Ulvestad et al. 2007). There is also much more tentative evidence for MBHs in other globular clusters (Maccarone et al. 2007; Trenti 2006; McLaughlin et al. 2006a; van den Bosch et al. 2006).

In summary, we find six additional galaxies that have both NCs and MBHs, of which four have measured black hole masses.

\section{DISCUSSION}

We now discuss the most interesting results from our study. These include the demographics of galaxies with both AGNs and NCs and the relative masses of MBHs and NCs. We also discuss our results in the context of models of the formation of $\mathrm{NCs}$ and MBHs.

\subsection{Demographics of Galaxies with AGNs and Nuclear Star Clusters}

In this section we first discuss the range of properties spanned by the galaxies with both NCs and AGNs and then examine the detailed demographics of these galaxies in our spectroscopic sample.

The left panels of Figure 5 show the Hubble type, mass, and absolute $B$-band magnitude of all galaxies presented in this paper 


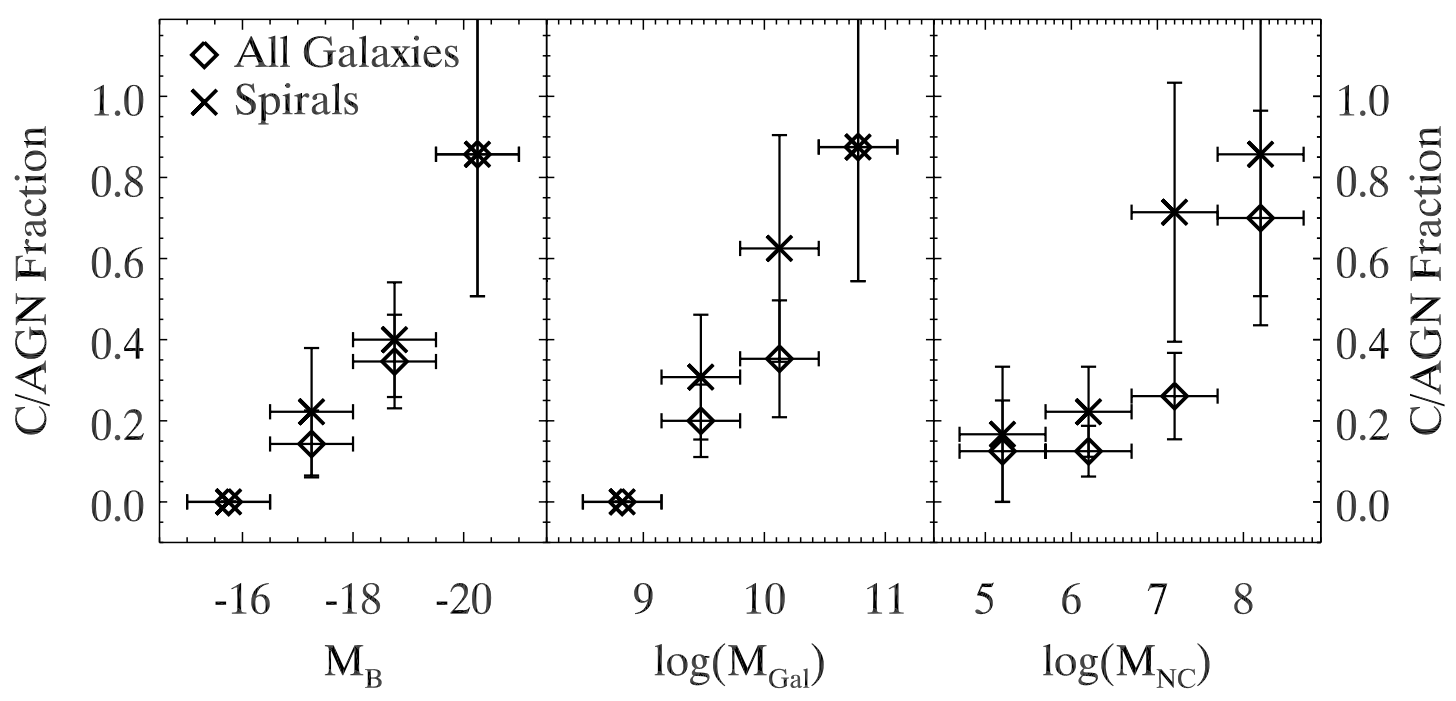

FIG. 6.-Fraction of the 75 galaxies in the spectroscopic sample classified as composite or AGN objects as a function of galaxy $B$-band absolute magnitude (left), galaxy mass (middle), and NC mass (right). In all panels, diamonds indicate the detection rate for all galaxies, while crosses include only the spiral galaxies ( $\mathrm{T}>0$ ). The vertical bars show Poisson errors, while the horizontal bars indicate the width of each bin.

hosting both NCs and candidate AGNs, including sources from the literature $(\S 4)$. Galaxies with both AGNs and NCs cross all Hubble types and reach magnitudes as faint as $M_{B} \sim-16$ and galaxy masses as low as $\sim 10^{9} M_{\odot}$. The AGN+NC galaxies are most common among early-type spirals and brighter/more massive galaxies (e.g., Decarli et al. 2007). As discussed in $\S 3.3$, the sources most likely to be $\mathrm{MBH}+\mathrm{NC}$ galaxies are the seven spectroscopic AGN candidates (Fig. 5, squares), the four brightest localized X-ray sources (black crosses), and the six sources from the literature not contained within our sample (circles). Even these galaxies span a wide range of Hubble types and luminosities/ masses. Thus, the overlap of MBHs and NCs is a phenomenon that occurs in all types of galaxies between masses of $10^{9}$ and $10^{11} M_{\odot}$

To be more quantitative about which galaxies host AGNs and which do not, we limit ourselves to the 75 galaxies in the spectroscopic sample for which both the detections and nondetections of AGNs are known. The right panels of Figure 5 show the classification of all galaxies in the spectroscopic sample; triangles represent galaxies with absorption spectra, crosses the star-forming galaxies, diamonds the composite galaxies, and squares the AGNs. Seven galaxies are classified as AGN (9\% of the sample), and 18 as composite or AGN ( $24 \%$ of the sample). Based on our discussion in $\S 3.3$, this suggests that $\gtrsim 10 \%$ of NCs in our spectroscopic sample host MBHs.

Most of the galaxies with AGN spectra (including the composite objects) are among the brighter and more massive galaxies in our sample. This trend is more clearly seen in Figure 6, which shows the fraction of galaxies in the spectroscopic sample with $\mathrm{C} / \mathrm{AGN}$ spectra as a function of galaxy $B$-band absolute magnitude, galaxy mass, and $\mathrm{NC}$ mass. A strong correlation is seen in each case, with the highest mass galaxies and NCs having AGN fractions of $\sim 80 \%$. This trend of increasing AGN activity with increasing galaxy mass is well documented (e.g., Ho et al. 1997a; Kauffmann et al. 2003; Decarli et al. 2007). The high detection rate of AGNs in galaxies with $M_{B}<-20$ and with galaxy mass of $\sim 10^{11} M_{\odot}$ is consistent with the findings of these previous surveys. In particular, Decarli et al. (2007) find that almost all Virgo Cluster spiral galaxies with dynamical mass $>10^{11} M_{\odot}$ have AGN spectra, with little dependence on Hubble type. However, a direct comparison with previous studies is complicated by the strong distance dependence of the detected AGN fraction (Kauffmann et al. 2003) and varying survey depths.

One way of considering whether NCs have any effect on the presence of an AGN is to look at the fraction of AGNs as a function of the galaxies' $M_{\mathrm{NC}} / M_{\text {gal }}$ (see Fig. 2). For galaxies with $M_{\mathrm{NC}} / M_{\text {gal }}>10^{-3}$ the fraction of AGNs is very similar $(28 \%)$ to those with $M_{\mathrm{NC}} / M_{\mathrm{gal}}<10^{-3}(25 \%)$. This indicates that the trend observed in the right panel of Figure 6 of increasing AGN fraction with increasing $\mathrm{NC}$ mass may just reflect the correlation of NC mass with galaxy mass.

Some variation in the AGN fraction is also seen with Hubble type. Ho et al. (1997a) find that transition and AGN spectra are found in $\sim 50 \%$ of E and S0 galaxies, $70 \%$ of Sa galaxies, $50 \%$ of $\mathrm{Sb}$ galaxies, and $15 \%$ of $\mathrm{Sc}-\mathrm{Sm}$ galaxies. Unfortunately, direct comparison to our sample is complicated by our inclusion of SDSS data, which differ from the HFS97 data in selection function and sensitivity. Taking just the data from their survey (Fig. 5, blue and green symbols), we classify one of four E/S0 galaxies, seven of nine $\mathrm{Sa}-\mathrm{Sbc}$ galaxies, and 3 of $10 \mathrm{Sc}-\mathrm{Sm}$ galaxies as $\mathrm{C} / \mathrm{AGN}$ galaxies. Despite the small number of galaxies (and slight differences in the classifications), these detection fractions in our galaxies with NCs are statistically consistent with what Ho et al. (1997a) find for the survey as a whole.

The spectroscopic sample does not uniformly cover our full NC galaxy sample. Good SDSS coverage of the Virgo clusters provides spectra for a high fraction of ellipticals $(\sim 75 \%)$, but the fraction of spirals with spectra is much lower $(\sim 30 \%)$ and is particularly lacking for the fainter spirals. This lack of spectra hinders our ability to determine the demographic trends of AGNs in our sample.

Overall, the demographic evidence suggests that galaxies with NCs have AGN fractions consistent with the population of galaxies as a whole. However, a larger, uniform sample of spectra for galaxies with and without NCs is needed to test this conclusion.

\subsection{The Relative Mass of Nuclear Star Clusters and Massive Black Holes}

In Ferrarese et al. (2006) the $\log M-\sigma$ relationships for NCs and MBHs have similar slopes, but the normalizations differ by roughly an order of magnitude, with NCs being more massive at 


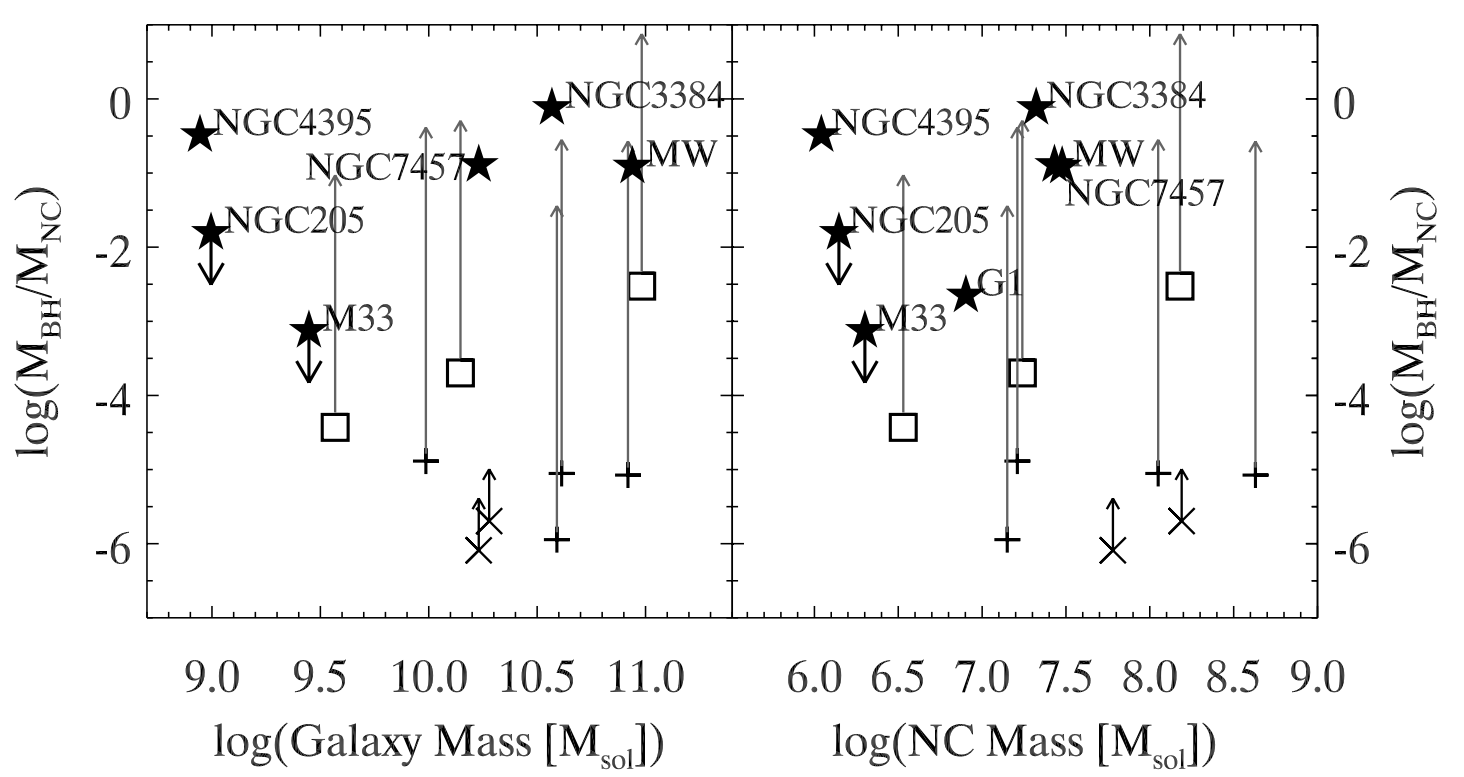

FIG. 7. - Limits on the relative mass of NCs and MBHs as a function of galaxy mass (left $)$ and NC mass (right). Stars are galaxies that have measured black hole masses (or upper limits) in the literature $(\S 3.3)$. The rest of the points represent lower limits based on the bolometric luminosity of their AGN. Different symbols indicate Seyfert galaxies (squares), LINERs ( plus signs), and X-ray sources (crosses). For the Seyfert galaxies and LINERs, the arrow length indicates the typical $L_{\mathrm{bol}} / L_{\mathrm{Edd}}$ for each class from Ho (2004).

a given bulge velocity dispersion. Similarly, Rossa et al. (2006) find that the normalization of the $M_{\mathrm{NC}}-L_{\text {bulge }, B}$ relation gives $\mathrm{NC}$ masses $\sim 3.3$ times more massive than the corresponding $\mathrm{MBH}$ relation from Marconi \& Hunt (2003). Based on a model for the $M_{\mathrm{CMO}}-M_{\mathrm{gal}}$ relation resulting from feedback of the $\mathrm{CMO}$ on the host galaxy, McLaughlin et al. (2006b) suggested that this offset may be the result of the reduced efficiency of $\mathrm{NC}$ formation feedback relative to feedback from an accreting $\mathrm{MBH}$, thus allowing the NCs to grow larger than an $\mathrm{MBH}$ at a given galaxy mass. With the present sample of NCs with AGN activity, we have the opportunity to potentially measure the relative mass of the NCs and MBHs within the same galaxies.

For four spiral galaxies from the literature (Milky Way, NGC 4395, NGC 3384, NGC 7457; see $\S 4$ ), we can combine direct estimates of the black hole masses and our own estimates of the NC masses ( $(2.1)$. Figure 7 shows the galaxy mass versus $M_{\mathrm{BH}} /$ $M_{\mathrm{NC}}$ ratios for these objects, which all fall between 0.1 and 1 .

We can constrain the $M_{\mathrm{BH}} / M_{\mathrm{NC}}$ ratio from our sample galaxies by deriving lower limits to the masses of black holes from the bolometric luminosity of the AGNs. Both the $\mathrm{H} \alpha$ and [O III] luminosities are known to be good indicators of the X-ray and bolometric luminosities of low-luminosity AGNs of all types (Ho et al. 2001), including sources with significant absorption in the X-ray (Panessa et al. 2006). Because the $\mathrm{H} \alpha$ luminosity suffers more contamination from star formation, we use [O III] luminosities to estimate $L_{\mathrm{X}}$ using the Panessa et al. (2006) relation:

$$
\log L_{\mathrm{X}}=1.22 \log L_{[\mathrm{O}}{ }_{\text {III }]}-7.34 \text {. }
$$

This yields typical $L_{[\mathrm{O} \text { III }} / L_{\mathrm{X}} \sim 15$ for our sample. We note that in the five cases in our sample where both X-ray data and C/AGN optical spectra are available, their luminosities are consistent with galaxies in the Panessa et al. (2006) sample. In these cases we derive the intrinsic X-ray luminosity based on the observed [O III] luminosity to correct for any X-ray absorption. Then, for both our optical and X-ray sources, we assume an $L_{\text {bol }} / L_{\mathrm{X}} \sim 10$ based on the results of Ho (1999) for a sample of seven low- luminosity AGNs. Note that this is a factor of $\sim 3$ less than the typically assumed $L_{\mathrm{bol}} / L_{\mathrm{X}}$ based on the luminosity function of luminous quasars (Elvis et al. 1994). The derived bolometric luminosities were then divided by the Eddington luminosity for a $1 M_{\odot}$ object to obtain a lower limit on the black hole mass. The resulting lower limits on $M_{\mathrm{BH}} / M_{\mathrm{NC}}$ [actually $\left(L_{\mathrm{bol}} / L_{\mathrm{Edd}, \odot}\right) / M_{\mathrm{NC}}$ ] are shown in Figure 7 for the nine strongest AGN candidates in our sample.

The highest lower limit from the sample is observed for NGC 6951 , which has $M_{\mathrm{BH}} / M_{\mathrm{NC}}>3 \times 10^{-3}$. The mean lower limit on $M_{\mathrm{BH}} / M_{\mathrm{NC}}$ is $3 \times 10^{-4}$ for the three Seyfert galaxies (squares) and $6 \times 10^{-6}$ for the four LINERs (crosses). For AGNs in general (i.e., without respect to the presence of an NC), the mean $L_{\mathrm{bol}} / L_{\mathrm{Edd}}$ ratios are $\sim 4 \times 10^{-4}$ for Seyfert galaxies and $\sim 3 \times 10^{-5}$ for LINERs and composite objects (Ho 2004). If these ratios hold for the AGNs in our sample, then our results are consistent with $M_{\mathrm{BH}} / M_{\mathrm{NC}}$ of near unity. These mean $L_{\mathrm{bol}} / L_{\mathrm{Edd}}$ ratios are indicated in Figure 7 by the length of the arrows from the Seyfert and LINER data points.

On the other hand, a strong piece of evidence that not all NCs host black holes of similar mass are the nondetections of black holes in Local Group galaxies with NCs. In M33, Gebhardt et al. (2001) place an upper limit on the black hole mass of $1500 M_{\odot}$. Assuming a mass for the M33 NC of $\sim 2 \times 10^{6} M_{\odot}$ (Kormendy $\&$ McClure 1993), this nondetection implies an $M_{\mathrm{BH}} / M_{\mathrm{NC}} \lesssim 10^{-3}$. Similarly, in NGC 205, the upper limit on the black hole mass is $2.2 \times 10^{4} M_{\odot}$ (Valluri et al. 2005), while the NC mass is $1.4 \times$ $10^{6} M_{\odot}$ (De Rijcke et al. 2006), giving $M_{\mathrm{BH}} / M_{\mathrm{NC}}<1.6 \times 10^{-2}$.

Finally, the globular cluster G1 in Andromeda, proposed to be the stripped NC of a dwarf galaxy (Meylan et al. 2001), also has an available measurement of $M_{\mathrm{BH}} / M_{\mathrm{NC}}$. The globular cluster mass is $8 \times 10^{6} M_{\odot}$ (Baumgardt et al. 2003), and the black hole mass is $1.8 \times 10^{4} M_{\odot}$ (Gebhardt et al. 2005), giving $M_{\mathrm{BH}} / M_{\mathrm{NC}} \sim 2 \times 10^{-3}$.

In summary, for four galaxies with measured black hole masses, the ratio of $M_{\mathrm{BH}} / M_{\mathrm{NC}}$ ranges from 0.1 to 1.0. Candidate AGNs in our sample also have luminosities that imply an $M_{\mathrm{BH}} / M_{\mathrm{NC}}$ 
of near unity. However, the black hole in the globular cluster G1 and black hole mass upper limits in M33 and NGC 205 show that at least some NCs have much lower $M_{\mathrm{BH}} / M_{\mathrm{NC}}$ or may not contain an $\mathrm{MBH}$ at all. The higher $M_{\mathrm{BH}} / M_{\mathrm{NC}}$ measurements may result from a bias toward finding high-mass black holes in galaxies that have AGNs or measurable black hole masses. Overall, the evidence presented here suggests that a wide range exists in the relative masses of $\mathrm{NCs}$ and $\mathrm{MBHs}$.

\subsection{Formation Mechanisms}

Based on the scaling relations for central massive objects and the presence of galaxies with both NCs and MBHs, there are two possibilities for the relative formation of central massive objects. Either the formation of MBHs could be directly linked in some way to the formation of NCs, or NCs and MBHs could be produced by processes that scale similarly with galaxy mass but are otherwise unrelated. We explore the first possibility below.

Because NCs are the dominant objects at the centers of lower mass galaxies (Ferrarese et al. 2006; Wehner \& Harris 2006), one natural possibility in hierarchical galaxy formation scenarios is that $\mathrm{NCs}$ lead to the formation of MBHs. Formation mechanisms for NCs fall into two classes: those that create NCs from the merging of globular clusters due to dynamical friction (Tremaine et al. 1975; Lotz et al. 2001), and those in which NCs are created in situ from gas accretion onto the nucleus due to galaxy merging (Mihos \& Hernquist 1994) or from disk gas dynamics (Milosavljević 2004; Bekki et al. 2006). The in situ scenario is favored by observations that NCs in spiral galaxies have complicated star formation histories, suggesting frequent episodic star formation (Walcher et al. 2006; Rossa et al. 2006). In addition, Seth et al. (2006) found NCs in late-type spirals that have young stellar disks aligned with the host galaxy disks, indicative of gas accretion onto the nucleus. Côté et al. (2006) also found support for gas accretion in the formation of NCs in elliptical galaxies; their more massive NCs are redder (implying higher metallicity) and more luminous than would be expected if they formed by mergers of globular clusters.

During in situ formation, $\mathrm{MBH}$ formation could occur through the merging of massive stars at the center of the cluster (see review by Miller \& Colbert 2004). For instance, dynamical modeling by Portegies Zwart et al. (2004) of the dense $3.5 \times 10^{5} M_{\odot}$ cluster MGG 11 in M82 shows that runaway collisions of massive stars lead to the formation of a black hole with mass $\sim 0.2 \%-1 \%$ of the mass of the cluster. Star formation episodes in NCs could certainly reproduce the high densities and massive star formation required for this mode of $\mathrm{MBH}$ formation. However, the MBHs in at least some of the NCs in our sample have masses nearly equal to that of the $\mathrm{NC}$; further accretion onto the $\mathrm{MBH}$ would be required to explain these cases. Also, if $\mathrm{MBHs}$ were a natural consequence of $\mathrm{NC}$ formation, we might expect to see a higher fraction of AGNs in galaxies with NCs than for the general galaxy population; this does not appear to be the case (see $\S 5.1$ ). These lines of evidence suggest that if there is a link between formation of NCs and MBHs, the NCs provide at most seed black holes that can then accrete into a more massive black hole.

The lack of NCs in high-mass galaxies also provides a clue to the evolution of CMOs. It is possible that during the buildup of more massive galaxies, the NCs could be destroyed (e.g., by black hole merging; Milosavljević \& Merritt 2001), leaving behind only an $\mathrm{MBH}$. Alternatively, the presence of an $\mathrm{MBH}$ would likely suppress the process of accretion and star formation in the NC due to enhanced feedback, thus preventing further NC growth.
Theoretical work and simulations are necessary to better understand these processes. Specifically, further simulations of $\mathrm{MBH}$ formation in massive clusters are needed that incorporate the episodic gas accretion thought to occur in NCs. Modeling of $\mathrm{NCs}$ and MBHs in hierarchical merging scenarios is also essential to explain the demographics of galaxies that host NCs and/or MBHs.

\section{CONCLUSIONS AND FUTURE WORK}

We have assembled a sample of 176 galaxies known to host NCs to study the relationship between NCs and MBHs. We then use optical spectroscopy and radio and X-ray data to look for AGNs in this sample. For 75 galaxies with available optical spectra, we classify 7 galaxies as AGNs and 11 as composite objects. X-ray catalogs from Chandra, ROSAT, and XMM-Newton provide detections of 22 galaxies in the sample, 4 of which have well-localized sources with X-ray luminosities indicating that they are likely AGNs. Lastly, we have assembled previously published results for nine galaxies that indicate that they have both NCs and MBHs/AGNs; three are included in our sample.

From this work we conclude that galaxies with both NCs and MBHs are relatively common. In addition, by examining the objects in our sample we find the following:

1. Galaxies that host both NCs and AGNs/MBHs span all Hubble types and have magnitudes as faint as $M_{B} \sim-16$ and masses as small as $10^{9} M_{\odot}$. For galaxies in our sample with available spectra, $\gtrsim 10 \%$ appear to host both NCs and MBHs.

2. Galaxies with NCs have AGN detection fractions that increase strongly with increasing galaxy and $\mathrm{NC}$ mass, consistent with previous studies of the general galaxy population. Variation of the AGN fraction with Hubble type in our sample is also consistent with the full Palomar survey (Ho et al. 1997a). This suggests that nuclear clusters do not play a strong role in promoting or limiting the activity of MBHs.

3. In four galaxies with NCs and measured black hole masses the ratios of the MBH mass to the NC mass are between 0.1 and 1. The luminosities of the AGNs in our sample of galaxies with NCs are also consistent with these mass ratios. However, the nondetection and low masses of MBHs in Local Group NCs suggest a much wider range of $\mathrm{MBH}-$ to-NC mass ratios.

4. For galaxies of the same mass, NCs in late-type spiral galaxies are typically an order of magnitude less massive than those in elliptical galaxies. This result will be discussed further in a forthcoming paper.

Although the data presented here provide compelling evidence for a significant overlap between the $\mathrm{MBH}$ and $\mathrm{NC}$ population, the evidence for the presence of an active $\mathrm{MBH}$ in many of the individual objects is quite weak. A number of observations could strengthen this evidence, including high-resolution Chandra observations or Spitzer mid-IR spectroscopy (e.g., Satyapal et al. 2007). Furthermore, less than half of the galaxies in our sample have available optical spectroscopy; obtaining spectroscopy for more is desirable and will help fill gaps in parameter space (e.g., the lack of spectra of low-mass early-type spirals). Finally, detailed studies of objects with both NCs and MBHs will be required to improve our understanding of the connections between these central massive objects.

The authors thank Christy Tremonti for providing significant assistance with the SDSS spectra. We also acknowledge valuable advice received from Margaret Geller, Julianne Dalcanton, 
David Helfand, Beth Willman, Andrew West, Nelson Caldwell, Andrés Jordán, Bob Becker, Ramesh Narayan, Martin Elvis, and Giuseppina Fabbiano. Anil Seth gratefully acknowledges the support of the CfA Postdoctoral Fellowship. Marcel Agüeros is supported by an NSF Astronomy and Astrophysics Postdoctoral Fellowship under award AST 06-02099.
This work would not have been possible without use of the Sloan Digital Sky Survey (http://www.sdss.org/), the NASA Extragalactic Database (http://nedwww.ipac.caltech.edu/), HyperLeda (http://leda.univ-lyon1.fr/), and NASA's High Energy Astrophysics Science Archive Research Center (http://heasarc .gsfc.nasa.gov/).

\section{REFERENCES}

Adelman-McCarthy, J. K., et al. 2007, ApJS, submitted (arXiv:0707.3413) Baldwin, J. A., Phillips, M. M., \& Terlevich, R. 1981, PASP, 93, 5

Baumgardt, H., Makino, J., Hut, P., McMillan, S., \& Portegies Zwart, S. 2003, ApJ, 589, L25

Becker, R. H., et al. 1995, ApJ, 450, 559

Becklin, E. E., \& Neugebauer, G. 1968, ApJ, 151, 145

Bedin, L. R., Piotto, G., Anderson, J., Cassisi, S., King, I. R., Momany, Y., \& Carraro, G. 2004, ApJ, 605, L125

Bekki, K., Couch, W. J., \& Shioya, Y. 2006, ApJ, 642, L133

Bell, E. F., McIntosh, D. H., Katz, N., \& Weinberg, M. D. 2003, ApJS, 149, 289 Best, P. N. 2004, MNRAS, 351, 70

Böker, T., Laine, S., van der Marel, R. P., Sarzi, M., Rix, H.-W., Ho, L. C., \& Shields, J. C. 2002, AJ, 123, 1389

Böker, T., Sarzi, M., McLaughlin, D. E., van der Marel, R. P., Rix, H.-W., Ho, L. C., \& Shields, J. C. 2004, AJ, 127, 105

Bruzual, G., \& Charlot, S. 2003, MNRAS, 344, 1000

Carollo, C. M., Stiavelli, M., de Zeeuw, P. T., \& Mack, J. 1997, AJ, 114, 2366

Carollo, C. M., Stiavelli, M., \& Mack, J. 1998, AJ, 116, 68

Carollo, C. M., Stiavelli, M., Seigar, M., de Zeeuw, P. T., \& Dejonghe, H. 2002, AJ, 123, 159

Colina, L., Gonzalez Delgado, R., Mas-Hesse, J. M., \& Leitherer, C. 2002, ApJ, 579, 545

Condon, J. J., et al. 1998, AJ, 115, 1693

Côté, P., et al. 2006, ApJS, 165, 57

Croft, S., de Vries, W., \& Becker, R. H. 2007, ApJ, 667, L13

Decarli, R., Gavazzi, G., Arosio, I., Cortese, L., Boselli, A., Bonfanti, C., \& Colpi, M. 2007, MNRAS, 381, 136

De Rijcke, S., Prugniel, P., Simien, F., \& Dejonghe, H. 2006, MNRAS, 369, 1321

Dubus, G., Charles, P. A., \& Long, K. S. 2004, A\&A, 425, 95

Dudik, R. P., Satyapal, S., Gliozzi, M., \& Sambruna, R. M. 2005, ApJ, 620, 113

Efron, B. 1982, The Jackknife, the Bootstrap and Other Resampling Plans (Philadelphia: SIAM)

Elvis, M., et al. 1994, ApJS, 95, 1

Fabbiano, G. 2006, ARA\&A, 44, 323

Ferrarese, L., et al. 2006, ApJ, 644, L21

Filippenko, A. V., \& Ho, L. C. 2003, ApJ, 588, L13

Filippenko, A. V., \& Sargent, W. L. W. 1985, ApJS, 57, 503 1989, ApJ, 342, L11

Flesch, E., \& Hardcastle, M. J. 2004, A\&A, 427, 387

Gebhardt, K., Rich, R. M., \& Ho, L. C. 2002, ApJ, 578, L41 2005, ApJ, 634, 1093

Gebhardt, K., et al. 2001, AJ, 122, 2469

Genzel, R., Thatte, N., Krabbe, A., Kroker, H., \& Tacconi-Garman, L. E. 1996, ApJ, 472, 153

Ghez, A. M., Salim, S., Hornstein, S. D., Tanner, A., Lu, J. R., Morris, M., Becklin, E. E., \& Duchêne, G. 2005, ApJ, 620, 744

Ghosh, K. K., Suleymanov, V., Bikmaev, I., Shimansky, S., \& Sakhibullin, N. 2006, MNRAS, 371, 1587

Gonzalez Delgado, R. M., Perez, E., Cid Fernandes, R., \& Schmitt, H. 2008, $\mathrm{AJ}$, in press (arXiv:0710.4450)

González-Martín, O., Masegosa, J., Márquez, I., Guerrero, M. A., \& DultzinHacyan, D. 2006, A\&A, 460, 45

Graham, A. W., \& Driver, S. P. 2007, ApJ, 655, 77

Graham, A. W., \& Guzmán, R. 2003, AJ, 125, 2936

Greene, J. E., \& Ho, L. C. 2007, ApJ, 667, 131

Haberl, F., et al. 2000, A\&AS, 142, 41

Ho, L. C. 1999, ApJ, 516, 672

Ho, L. C., Filippenko, A. V., \& Sargent, W. L. W. 1997a, ApJ, 487, 568 1997b, ApJS, 112, 315 (HFS97)

Ho, L. C., Terashima, Y., \& Ulvestad, J. S. 2003, ApJ, 589, 783

Ho, L. C., et al. 2001, ApJ, 549, L51

Ho, L. C. W. 2004, in Coevolution of Black Holes and Galaxies, ed. L. C. Ho (Cambridge: Cambridge Univ. Press), 292

Jensen, J. B., Tonry, J. L., Barris, B. J., Thompson, R. I., Liu, M. C., Rieke, M. J., Ajhar, E. A., \& Blakeslee, J. P. 2003, ApJ, 583, 712

Jones, D. H., et al. 2004, MNRAS, 355, 747

Kauffmann, G., et al. 2003, MNRAS, 346, 1055
Kennicutt, R. C., Jr. 1998, ApJ, 498, 541

Kewley, L. J., Dopita, M. A., Sutherland, R. S., Heisler, C. A., \& Trevena, J. 2001, ApJ, 556, 121

Kewley, L. J., Groves, B., Kauffmann, G., \& Heckman, T. 2006, MNRAS, 372, 961

Kormendy, J., \& McClure, R. D. 1993, AJ, 105, 1793

Li, Y., Haiman, Z., \& Mac Low, M.-M. 2007, ApJ, 663, 61

Lotz, J. M., Telford, R., Ferguson, H. C., Miller, B. W., Stiavelli, M., \& Mack, J. 2001, ApJ, 552, 572

Maccarone, T. J., Kundu, A., Zepf, S. E., \& Rhode, K. L. 2007, Nature, 445, 183

Maoz, D., Nagar, N. M., Falcke, H., \& Wilson, A. S. 2005, ApJ, 625, 699

Marconi, A., \& Hunt, L. K. 2003, ApJ, 589, L21

Matthews, L. D., et al. 1999, AJ, 118, 208

McLaughlin, D. E., Anderson, J., Meylan, G., Gebhardt, K., Pryor, C., Minniti, D., \& Phinney, S. 2006a, ApJS, 166, 249

McLaughlin, D. E., King, A. R., \& Nayakshin, S. 2006b, ApJ, 650, L37

Mei, S., et al. 2007, ApJ, 655, 144

Meylan, G., Sarajedini, A., Jablonka, P., Djorgovski, S. G., Bridges, T., \& Rich, R. M. 2001, AJ, 122, 830

Mickaelian, A. M., et al. 2006, A\&A, 449, 425

Mihos, J. C., \& Hernquist, L. 1994, ApJ, 437, L47

Miller, M. C., \& Colbert, E. J. M. 2004, Int. J. Mod. Phys. D, 13, 1

Milosavljević, M. 2004, ApJ, 605, L13

Milosavljević, M., \& Merritt, D. 2001, ApJ, 563, 34

Moran, E. C., Halpern, J. P., \& Helfand, D. J. 1996, ApJS, 106, 341

Mukai, K. 1993, Legacy, 3, 21

Nagar, N. M., Falcke, H., \& Wilson, A. S. 2005, A\&A, 435, 521

Narayan, R., Mahadevan, R., Grindlay, J. E., Popham, R. G., \& Gammie, C. 1998, ApJ, 492, 554

Noyola, E., Gebhardt, K., \& Bergmann, M. 2006, in ASP Conf. Ser. 352, New Horizons in Astronomy: Frank N. Bash Symposium, ed. S. J. Kannappan et al. (San Francisco: ASP), 269

Osterbrock, D. E. 1989, Astrophysics of Gaseous Nebulae and Active Galactic Nuclei (Mill Valley: University Science Books)

Panessa, F., Bassani, L., Cappi, M., Dadina, M., Barcons, X., Carrera, F. J., Ho, L. C., \& Iwasawa, K. 2006, A\&A, 455, 173

Panzera, M. R., et al. 2003, A\&A, 399, 351

Paturel, G., Theureau, G., Bottinelli, L., Gouguenheim, L., Coudreau-Durand, N., Hallet, N., \& Petit, C. 2003, A\&A, 412, 57

Peterson, B. M., et al. 2005, ApJ, 632, 799

Pooley, D., \& Rappaport, S. 2006, ApJ, 644, L45

Portegies Zwart, S. F., Baumgardt, H., Hut, P., Makino, J., \& McMillan, S. L. W. 2004, Nature, 428, 724

Ptak, A., \& Griffiths, R. 2003, in ASP Conf. Ser. 295, Astronomical Data Analysis Software and Systems XII, ed. H. E. Payne, R. I. Jedrzejewski, \& R. N. Hook (San Francisco: ASP), 465

Rasio, F. A., et al. 2006, preprint (astro-ph/0611615)

Ravindranath, S., Ho, L. C., Peng, C. Y., Filippenko, A. V., \& Sargent, W. L. W. 2001, AJ, 122, 653

Rossa, J., van der Marel, R. P., Böker, T., Gerssen, J., Ho, L. C., Rix, H.-W., Shields, J. C., \& Walcher, C.-J. 2006, AJ, 132, 1074

Satyapal, S., Vega, D., Heckman, T., O'Halloran, B., \& Dudik, R. 2007, ApJ, 663, L9

Scarlata, C., et al. 2004, AJ, 128, 1124

Schödel, R., et al. 2007, A\&A, 469, 125

Seth, A. C., Dalcanton, J. J., Hodge, P. W., \& Debattista, V. P. 2006, AJ, 132, 2539

Shapley, A., Fabbiano, G., \& Eskridge, P. B. 2001, ApJS, 137, 139

Shields, J., Walcher, C. J. M., Boeker, T., Ho, L. C., Rix, H.-W., \& van der Marel, R. P. 2008, ApJ, submitted

Shields, J. C., et al. 2007, ApJ, 654, 125

Sivakoff, G. R., et al. 2007, ApJ, 660, 1246

Terashima, Y., \& Wilson, A. S. 2003, ApJ, 583, 145

Thim, F., Hoessel, J. G., Saha, A., Claver, J., Dolphin, A., \& Tammann, G. A. 2004, AJ, 127, 2322

Tonry, J. L., Dressler, A., Blakeslee, J. P., Ajhar, E. A., Fletcher, A. B., Luppino, G. A., Metzger, M. R., \& Moore, C. B. 2001, ApJ, 546, 681 
Tremaine, S., et al. 2002, ApJ, 574, 740

Tremaine, S. D., Ostriker, J. P., \& Spitzer, L. 1975, ApJ, 196, 407

Tremonti, C. A., et al. 2004, ApJ, 613, 898

Trenti, M. 2006, MNRAS, submitted (astro-ph/0612040)

Ulvestad, J. S., Greene, J. E., \& Ho, L. C. 2007, ApJ, 661, L151

Valluri, M., Ferrarese, L., Merritt, D., \& Joseph, C. L. 2005, ApJ, 628, 137

van den Bosch, R., de Zeeuw, T., Gebhardt, K., Noyola, E., \& van de Ven, G. 2006, ApJ, 641, 852

van der Marel, R. P. 2004, in Coevolution of Black Holes and Galaxies, ed. L. C. Ho (Cambridge: Cambridge Univ. Press), 37
Véron-Cetty, M.-P., et al. 2004, A\&A, 414, 487

Voges, W., et al. 1999, A\&A, 349, 389

Walcher, C. J., Böker, T., Charlot, S., Ho, L. C., Rix, H.-W., Rossa, J., Shields,

J. C., \& van der Marel, R. P. 2006, ApJ, 649, 692

Walcher, C. J., et al. 2005, ApJ, 618, 237

Wehner, E. H., \& Harris, W. E. 2006, ApJ, 644, L17

White, R. L., et al. 2007, ApJ, 654, 99

York, D. G., et al. 2000, AJ, 120, 1579

Yukita, M., Swartz, D. A., Soria, R., \& Tennant, A. F. 2007, ApJ, 664, 277 